NBER WORKING PAPER SERIES

\title{
ON THE CORRELATION STRUCTURE OF MICROSTRUCTURE NOISE: A FINANCIAL ECONOMIC APPROACH
}

\author{
Francis X. Diebold \\ Georg Strasser \\ Working Paper 16469 \\ http://www.nber.org/papers/w16469 \\ NATIONAL BUREAU OF ECONOMIC RESEARCH \\ 1050 Massachusetts Avenue \\ Cambridge, MA 02138 \\ October 2010
}

For comments and suggestions we thank Rob Engle, Peter Hansen, Charles Jones, Eugene Kandel, Gideon Saar, Frank Schorfheide, Enrique Sentana, and three anonymous referees. We are also grateful to participants at the NBER Conference on Market Microstructure, the Oxford-Man Institute Conference on the Financial Econometrics of Vast Data, and the Christmas Meeting of German Economists Abroad. We thank Ole E. Barndor-Nielsen, Peter R. Hansen, Asger Lunde and Neil Shephard for sharing their data with us. The views expressed herein are those of the authors and do not necessarily reflect the views of the National Bureau of Economic Research.

NBER working papers are circulated for discussion and comment purposes. They have not been peerreviewed or been subject to the review by the NBER Board of Directors that accompanies official NBER publications.

(C) 2010 by Francis X. Diebold and Georg Strasser. All rights reserved. Short sections of text, not to exceed two paragraphs, may be quoted without explicit permission provided that full credit, including (C) notice, is given to the source. 
On the Correlation Structure of Microstructure Noise: A Financial Economic Approach

Francis X. Diebold and Georg Strasser

NBER Working Paper No. 16469

October 2010

JEL No. C51,D82,D83,G14,G20

\begin{abstract}
$\underline{\text { ABSTRACT }}$
We introduce the financial economics of market microstructure into the financial econometrics of asset return volatility estimation. In particular, we use market microstructure theory to derive the crosscorrelation function between latent returns and market microstructure noise, which feature prominently in the recent volatility literature. The cross-correlation at zero displacement is typically negative, and cross-correlations at nonzero displacements are positive and decay geometrically. If market makers are sufficiently risk averse, however, the cross-correlation pattern is inverted. Our results are useful for assessing the validity of the frequently-assumed independence of latent price and microstructure noise, for explaining observed cross-correlation patterns, for predicting as-yet undiscovered patterns, and for making informed conjectures regarding improved volatility estimation methods.
\end{abstract}

Francis X. Diebold

Department of Economics

University of Pennsylvania

3718 Locust Walk

Philadelphia, PA 19104-6297

and NBER

fdiebold@sas.upenn.edu

Georg Strasser

Department of Economics

Boston College

140 Commonwealth Avenue

Chestnut Hill, MA 02467-3806

Georg.Strasser@bc.edu 


\section{Introduction}

Recent years have seen substantial progress in asset return volatility measurement, with important applications to asset pricing, portfolio allocation and risk management. In particular, so-called realized variances and covariances ("realized volatilities"), based on increasinglyavailable high-frequency data, have emerged as central for several reasons. ${ }^{1}$ They are, for example, largely model-free (in contrast to traditional model-based approaches such as GARCH or stochastic volatility), they are computationally trivial, and they are in principle highly accurate.

A tension arises, however, linked to the last of the above desiderata. Econometric theory suggests the desirability of sampling as often as possible to obtain highly accurate volatility estimates, but financial market reality suggests otherwise. In particular, microstructure noise (MSN), such as bid-ask bounce associated with ultra-high-frequency sampling, may contaminate the observed price, potentially rendering naively-calculated realized volatilities unreliable.

Early work (e.g., Andersen et al., 2001a b, 2003, Barndorff-Nielsen and Shephard, 2002a, b) addressed the sampling issue by attempting to sample often, but not "too often," implicitly or explicitly using the volatility signature plot of Andersen et al. (2000) to guide sampling frequency, typically resulting in use of five- to thirty-minute returns. ${ }^{2}$

Much higher-frequency data are usually available, however, so reducing the sampling frequency to insure against MSN discards potentially valuable information. To use all information, more recent work has emphasized MSN-robust realized volatilities that use returns sampled at very high frequencies. Examples include Zhang et al. (2005), Bandi and Russell (2008), Aï-Sahalia et al. (2010), Hansen and Lunde (2006), and Barndorff-Nielsen et al. 2008a, 2010). That literature is almost entirely statistical, however, which is unfortunate because it makes important assumptions regarding the nature of the latent price, the MSN, and their interaction, and purely statistical thinking offers little guidance. A central example concerns the interaction (if any) between latent price and MSN. Some authors such as Bandi and Russell assume no correlation (perhaps erroneously), whereas in contrast BarndorffNielsen et al. (2008a b) allow for correlation (perhaps unnecessarily).

To improve this situation, we explicitly recognize that MSN results from the strategic behavior of economic agents, and we push toward integration of the financial economics of market microstructure with the financial econometrics of volatility estimation. In particular,

\footnotetext{
${ }^{1}$ Several surveys are now available, ranging from the comparatively theoretical treatments of BarndorffNielsen and Shephard (2007) and Andersen et al. (2010) to the applied perspective of Andersen et al. (2006).

${ }^{2}$ The volatility signature plot shows average daily realized volatility as a function of underlying sampling frequency.
} 
we explore the implications of microstructure theory for the relationship between latent price and MSN, characterizing the cross-correlation structure between latent price and MSN, contemporaneously and dynamically, in a variety of leading environments, including those of Roll (1984), Glosten and Milgrom (1985), Kyle (1985), Easley and O'Hara (1992), and Hasbrouck $(2002) .^{3}$

We proceed as follows. In section 2 we introduce our general framework, which nests a variety of microstructure models. In sections 3 and 4 we provide detailed analyses of models of private information, distinguishing two types of latent prices based on the implied level of market efficiency. In particular, we treat strong form efficiency in section 3 and semi-strong form efficiency in section 4. We sketch additional econometric implications of our findings in section 5 , and we conclude in section 6 .

\section{The Framework}

We begin in section 2.1 by introducing our general framework relating latent prices, observed prices, and MSN in a wide range of market-making environments. We then provide, in section 2.2 , a generic (model-free) statistical result on the nature of correlation between latent price and MSN. Finally, in section 2.3 we introduce several workhorse microstructural economic models of market making, which we use heavily in subsequent analyses.

\subsection{Latent Prices, Observed Prices and Microstructure Noise}

Let $p_{t}^{*}$ denote the (logarithm of the) strong form efficient price of some asset in the calendar time period $t .{ }^{4}$ This price, strictly exogenously changing every $T^{t h}$-period and at time $t$ known only to the informed traders, follows the process ${ }^{5}$

$$
\begin{gathered}
p_{t}^{*}= \begin{cases}p_{t-1}^{*}+\sigma \varepsilon_{t}, & \forall t=\kappa T, \kappa \in \mathbb{Z} \\
p_{t-1}^{*}, & \text { otherwise }\end{cases} \\
\varepsilon_{t} \stackrel{\text { iid }}{\sim}(0,1) .
\end{gathered}
$$

\footnotetext{
${ }^{3}$ For insightful surveys of the key models, see O'Hara (1995) and Hasbrouck (2007).

${ }^{4} \mathrm{We}$ focus on the information conveyed by the trade direction only. Today's markets are dominated by orders split into many small trades, so that the size of individual orders is only minimally informative. Hence we focus on the trade direction as primary source of trade-related information.

${ }^{5}$ The setup we present here is a slight generalization of the "generalized Roll model" of Hasbrouck (2007). As common in the high-frequency literature, we drop the deterministic drift term, but our results hold for any nonzero deterministic drift, if price changes are defined net of drift.
} 
Order-splitting into many small trades has become dominant in recent years. In line with recent developments we consider in this paper the limiting case of all trades being of unit size. Under a suitable choice of calendar frequency, as illustrated by Figure 1, this allows us to map any trading pattern into calendar time with unit trades.

Figure 1: Mapping Tick-Time into Calendar Time

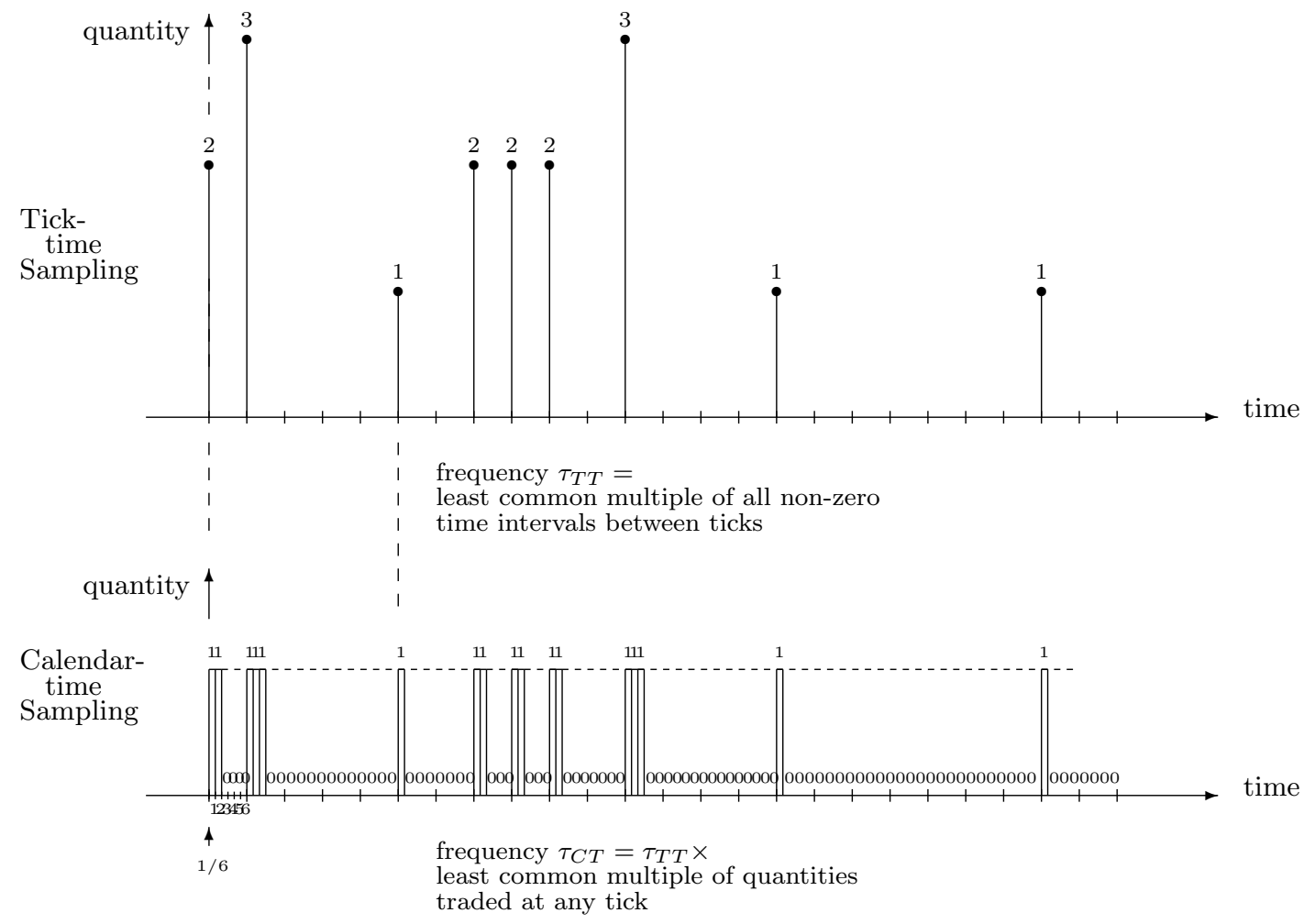

Let $q_{t}$ denote the direction of the trade in period $t$, where $q_{t}=+1$ denotes a buy, $q_{t}=-1$ a sell, and $q_{t}=0$ that no trade occurs in the period. Define $p_{t}^{e}$ as the expected efficient price directly before the trade occurs. The semi-strong form efficient price, which summarizes the knowledge of the market maker after the trade, ${ }^{6}$ is

$$
\tilde{p}_{t}^{e}=p_{t}^{e}+\lambda_{t} q_{t}
$$

where $\lambda_{t} \geq 0$ captures the response to asymmetric information revealed by the trade direction $q_{t}$. At the beginning of each trading round, additional information about $p_{t}^{*}$ and $\varepsilon_{t}$ might be revealed by information diffusion from other markets. This information, summarized by $\omega_{t}$,

\footnotetext{
${ }^{6}$ This terminology is borrowed from the asset pricing literature. In contrast to the strong form efficient price, which incorporates all public and private information, the semi-strong form efficient price only incorporates all publicly available information (Fama, 1970).
} 
makes the market maker revise his price expectation for the next period according to ${ }^{7}$

$$
p_{t}^{e}=\tilde{p}_{t-1}^{e}+\omega_{t}
$$

Assuming that the (logarithm of) price quotes are symmetric around the expected efficient price before the trade, ${ }^{8}$ the (logarithm of the) observed transaction price can be written as

$$
p_{t}=p_{t}^{e}+s_{t} q_{t}
$$

where $s_{t}$ is one-half of the spread. In particular, the bid price is $p_{t}^{b i d}=p_{t}^{e}-s_{t}$, the ask price is $p_{t}^{a s k}=p_{t}^{e}+s_{t}$, and the mid price is $p_{t}^{m i d}=p_{t}^{e}$. These prices and their relationships are illustrated by Figure 2 .

Figure 2: Timing of Information and Prices

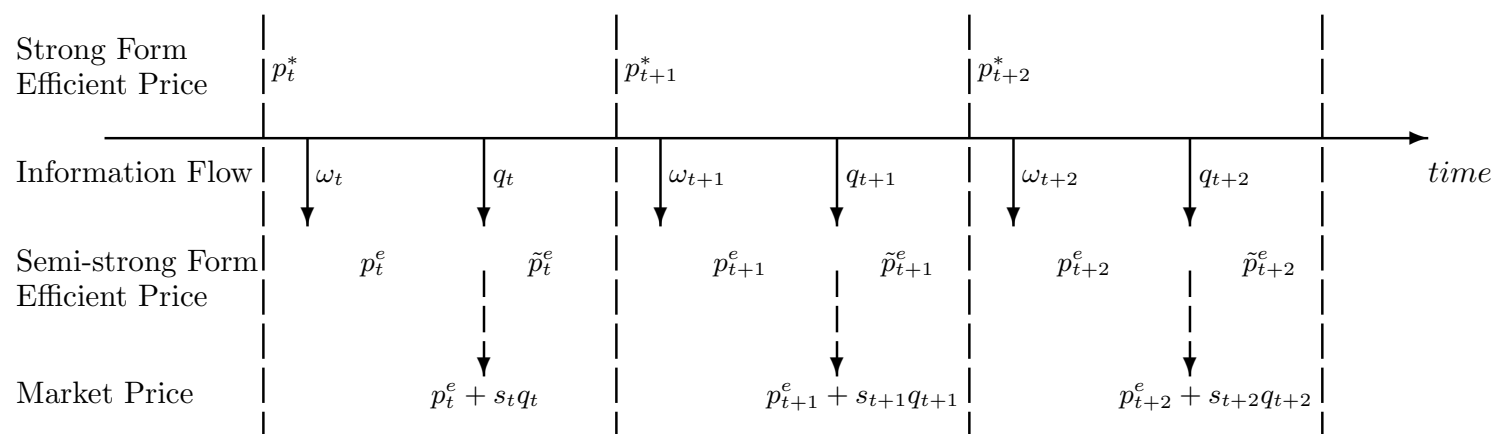

Strong form efficient returns in periods $t=\kappa T$ are therefore

$$
\Delta p_{t}^{*} \equiv p_{t}^{*}-p_{t-1}^{*}=\sigma \varepsilon_{t},
$$

and zero in all other periods. Semi-strong form efficient returns are

$$
\Delta \tilde{p}_{t}^{e} \equiv \tilde{p}_{t}^{e}-\tilde{p}_{t-1}^{e}=\lambda_{t} q_{t}+\omega_{t}
$$

and market returns are

$$
\begin{aligned}
\Delta p_{t} \equiv p_{t}-p_{t-1} & =\Delta p_{t}^{e}+s_{t} q_{t}-s_{t-1} q_{t-1} \\
& =\Delta \tilde{p}_{t}^{e}+\left(s_{t}-\lambda_{t}\right) q_{t}-\left(s_{t-1}-\lambda_{t-1}\right) q_{t-1} .
\end{aligned}
$$

\footnotetext{
${ }^{7}$ In periods in which $p_{t-1}^{*}$ becomes public information, this becomes $p_{t}^{e}=p_{t-1}^{*}+\omega_{t}$.

${ }^{8}$ We use the approximation $\ln (P+S)=\ln \left(P\left(1+\frac{S}{P}\right)\right)=p+\ln \left(1+\frac{S}{P}\right) \approx p+\frac{S}{P} \equiv p+s$, where $P$ and $S$ denote price and spread before taking the natural logarithm, respectively.
} 
We use the term "latent price" as a general term comprising both types of efficient prices. The two latent prices defined here are conceptually very distinct and appeal to distinct audiences. For example, on the one hand, a pure theorist may want to understand the properties of the full-information price, and is thus interested in an estimate of the volatility of the strong form efficient return (6). One the other hand, a market maker may need a volatility measure to calculate risk exposure, thus his relevant price for the asset is $\tilde{p}_{t}^{e}$, the price at which he keeps the asset on his accounts. It is the volatility of (7), and not of (6), that affects his balance sheet.

Microstructure noise (MSN) is the difference between the observed market return and the latent return. Depending on whether one considers the strong form efficient return or the semi-strong form efficient return, the noise is defined either as strong form noise

$$
\Delta u_{t} \equiv \Delta p_{t}-\Delta p_{t}^{*}
$$

or as semi-strong form noise ${ }^{9}$

$$
\Delta u_{t} \equiv \Delta p_{t}-\Delta \tilde{p}_{t}^{e}
$$

As we show in this paper, these two types of noise differ fundamentally in their crosscorrelation properties. It is therefore essential for a researcher to be clear in advance what type of latent price the object of interest is, because each type of efficiency requires different procedures to remove MSN appropriately.

A convenient estimator of the variance of the strong form efficient return, $\sigma^{2}$, is the realized variance Andersen et al. (2001b). Realized variance during the time interval [0,T] is defined as the sum of squared market returns over the interval, i.e. as

$$
\operatorname{Var}\left(\Delta p_{t}\right)=\sum_{t=1}^{T} \Delta p_{t}^{2}
$$

In the presence of MSN, the realized variance is generally a biased estimate of the variance of the efficient return, $\sigma^{2}$. To see this, decompose the noise into two components, i.e. $\Delta u_{t}=$ $\Delta u_{t}^{b a}+\Delta u_{t}^{a s y}$. The first component, $\Delta u_{t}^{b a}$, is assumed to be uncorrelated with the latent price of interest, reflecting for example the bid-ask bounce in a market populated with uninformed traders only. The second component, $\Delta u_{t}^{a s y}$, is correlated with the efficient price, and reflects for example the effect of asymmetric information. Realized variance can now be decomposed

\footnotetext{
${ }^{9}$ We assume throughout that market conditions are stable and that market prices $p_{t}$ adjust sufficiently fast so that the noise process $\Delta u_{t}$ is covariance stationary.
} 
- here shown for the strong form efficient price - as

$$
\begin{aligned}
\operatorname{Var}\left(\Delta p_{t}\right) & =\operatorname{Var}\left(\Delta p_{t}^{*}+\Delta u_{t}^{b a}+\Delta u_{t}^{a s y}\right) \\
& =\sigma^{2}+\operatorname{Var}\left(\Delta u_{t}^{b a}\right)+\operatorname{Var}\left(\Delta u_{t}^{a s y}\right)+2 \operatorname{Cov}\left(\Delta p_{t}^{*}, \Delta u_{t}^{a s y}\right)
\end{aligned}
$$

The bias of the realized variance can stem from any of the last three terms, which are all nonzero in general. Realized variance estimation under the independent noise assumption accounts for the second and third positive terms, but ignores the last term, which is typically negative (Hansen and Lunde, 2006). Correcting the estimates for independent noise only, always reduces the volatility estimate. But because such a correction ignores the last term, which is the second channel through which asymmetric information affects the realized variance estimate, the overall reduction might be too much. Further, serial correlation of noise, or equivalently a cross-correlation between noise and latent returns at nonzero displacement, requires the use of robust estimators for both the variance and the covariance terms. In this paper we determine what correlation and serial correlation market microstructure theory predicts, and how market microstructure theory can be useful for obtaining improved estimates of integrated variance.

\subsection{Statistical Characterization of Return/Noise Correlations}

We focus in this paper on the cross-correlation between latent returns and noise contemporaneously and at all displacements. Throughout, we refer to this quantity simply as the "cross-correlation".

Here we present a simple but important insight about the cross-correlation, true under very general conditions because it follows directly from the definition of covariance. In particular, for the price processes given by (1)-(5) the contemporaneous cross-correlation $\operatorname{Corr}\left(\Delta p_{t}^{*}, \Delta u_{t}\right)$ is positive only if the market return, $\Delta p_{t}$, is more volatile than the latent return. More precisely, for strong form efficient returns ${ }^{10}$

$$
\operatorname{Corr}\left(\Delta p_{t}^{*}, \Delta u_{t}\right)>0 \Leftrightarrow E\left(\Delta p_{t} \Delta p_{t}^{*}\right)>\operatorname{Var}\left(\Delta p_{t}^{*}\right) \Leftrightarrow \operatorname{Corr}\left(\Delta p_{t}, \Delta p_{t}^{*}\right)>\sqrt{\frac{\operatorname{Var}\left(\Delta p_{t}^{*}\right)}{\operatorname{Var}\left(\Delta p_{t}\right)}},
$$

\footnotetext{
${ }^{10}$ To see this for strong form efficient returns, simply note that the unconditional expectation of both microstructure noise and the latent price change is zero $\left(E\left(\Delta u_{t}\right)=0, E\left(\Delta p_{t}^{*}\right)=\frac{1}{T} \sigma E\left(\varepsilon_{t}\right)=0\right)$, so that the contemporaneous cross-covariance between strong form efficient returns and noise is

$$
\operatorname{Cov}\left(\Delta p_{t}^{*}, \Delta u_{t}\right)=E\left(\Delta p_{t}^{*}\left(\Delta p_{t}-\Delta p_{t}^{*}\right)\right)=E\left(\Delta p_{t}^{*} \Delta p_{t}\right)-\operatorname{Var}\left(\Delta p_{t}^{*}\right)
$$
}

The logic for semi-strong form efficient returns is analogous, because $E\left(\Delta \tilde{p}_{t}^{e}\right)=0$. 
and for semi-strong form efficient returns

$$
\operatorname{Corr}\left(\Delta \tilde{p}_{t}^{e}, \Delta u_{t}\right)>0 \Leftrightarrow E\left(\Delta p_{t} \Delta \tilde{p}_{t}^{e}\right)>\operatorname{Var}\left(\Delta \tilde{p}_{t}^{e}\right) \Leftrightarrow \operatorname{Corr}\left(\Delta p_{t}, \Delta \tilde{p}_{t}^{e}\right)>\sqrt{\frac{\operatorname{Var}\left(\Delta \tilde{p}_{t}^{e}\right)}{\operatorname{Var}\left(\Delta p_{t}\right)}}
$$

Cross-correlations at displacements $\tau \geq 1$ are positive if and only if the current market price responds stronger in the direction of a previous latent price change than the current latent price itself. More precisely, for strong form efficient returns ${ }^{11}$

$$
\operatorname{Corr}\left(\Delta p_{t-\tau}^{*}, \Delta u_{t}\right)>0 \Leftrightarrow E\left(\Delta p_{t} \Delta p_{t-\tau}^{*}\right)>0
$$

and for semi-strong form efficient returns

$$
\operatorname{Corr}\left(\Delta \tilde{p}_{t-\tau}^{e}, \Delta u_{t}\right)>0 \Leftrightarrow E\left(\Delta p_{t} \Delta \tilde{p}_{t-\tau}^{e}\right)>E\left(\Delta \tilde{p}_{t}^{e} \Delta \tilde{p}_{t-\tau}^{e}\right)
$$

The statistical insight offered here is helpful at a broad conceptual level - without referring to any specific model of market participants' behavior, we can nevertheless isolate the properties of $\Delta p_{t}$ that determine the cross-correlation pattern - but it offers no insight into the nature of cross-correlation patterns induced by strategic economic behavior. In the financial economic environments that will concern us, the properties of $\Delta p_{t}$, and hence the cross-correlation patterns, are determined by (1) the market microstructure, and (2) the market maker's loss function. Hence we introduce them now in some detail.

\subsection{Introducing Markets and Market Makers}

Whereas the strong form efficient price (1) is an exogenous stochastic process, the semi-strong form efficient price (3) and the market price (5) are an outcome of the market participants' optimizing behavior. As such the latter are not time series of unknown properties generated by a black box. Instead, key properties of the data generator - the financial market - are often observable and allow inferring properties of these price series. This is what we do in this paper.

Generally speaking, the market price depends on the information available about the

\footnotetext{
${ }^{11}$ These results follow again from the definition of covariance; the cross-covariance at nonzero displacements between latent returns $\tau \geq 1$ periods ago and noise is

$$
\operatorname{Cov}\left(\Delta p_{t-\tau}^{*}, \Delta u_{t}\right)=E\left(\Delta p_{t-\tau}^{*}\left(\Delta p_{t}-\Delta p_{t}^{*}\right)\right)=E\left(\Delta p_{t-\tau}^{*} \Delta p_{t}\right)-E\left(\Delta p_{t-\tau}^{*} \Delta p_{t}^{*}\right)
$$

Independence of strong form efficient price changes and non-forgetting immediately implies (10). The logic for semi-strong form efficient returns is again analogous, except that serial correlation of semi-strong form efficient returns, $\Delta p_{t}^{e}$, must be taken into account.
} 
strong form efficient price and the market participants' response to this information. The information process matters in two ways: First, via its information content, and second, via the time span $T$ in which it is not publicly known but valid. The price updating rule determines how, and how quickly, market prices respond to new information. Of particular importance is whether the market maker can quote prices dependent on the direction of trade, i.e. whether he is free to charge any spread, because direction-dependent quotes allow prices to react instantaneously.

We consider limit-order markets, populated by informed and uninformed traders. Market makers are the counterparty of all trades. Each trading round they quote price $p_{t}^{\text {mid }}$ and spread $s_{t}$ for one unit of the asset. Thereafter, as shown in figure 3, informed traders screen the market with probability $\alpha$ for profitable trading opportunities. They buy if $p_{t}^{*}>p_{t}^{a s k} \equiv p_{t}^{\text {mid }}+s_{t}$, sell if $p_{t}^{*}<p_{t}^{\text {bid }} \equiv p_{t}^{\text {mid }}-s_{t}$, and refuse to trade otherwise. In periods of no informed trade, uninformed traders trade instead with probability $\beta$, buying and selling with equal probability. ${ }^{12}$

Figure 3: Sequence of Informed and Uninformed Trading Decisions

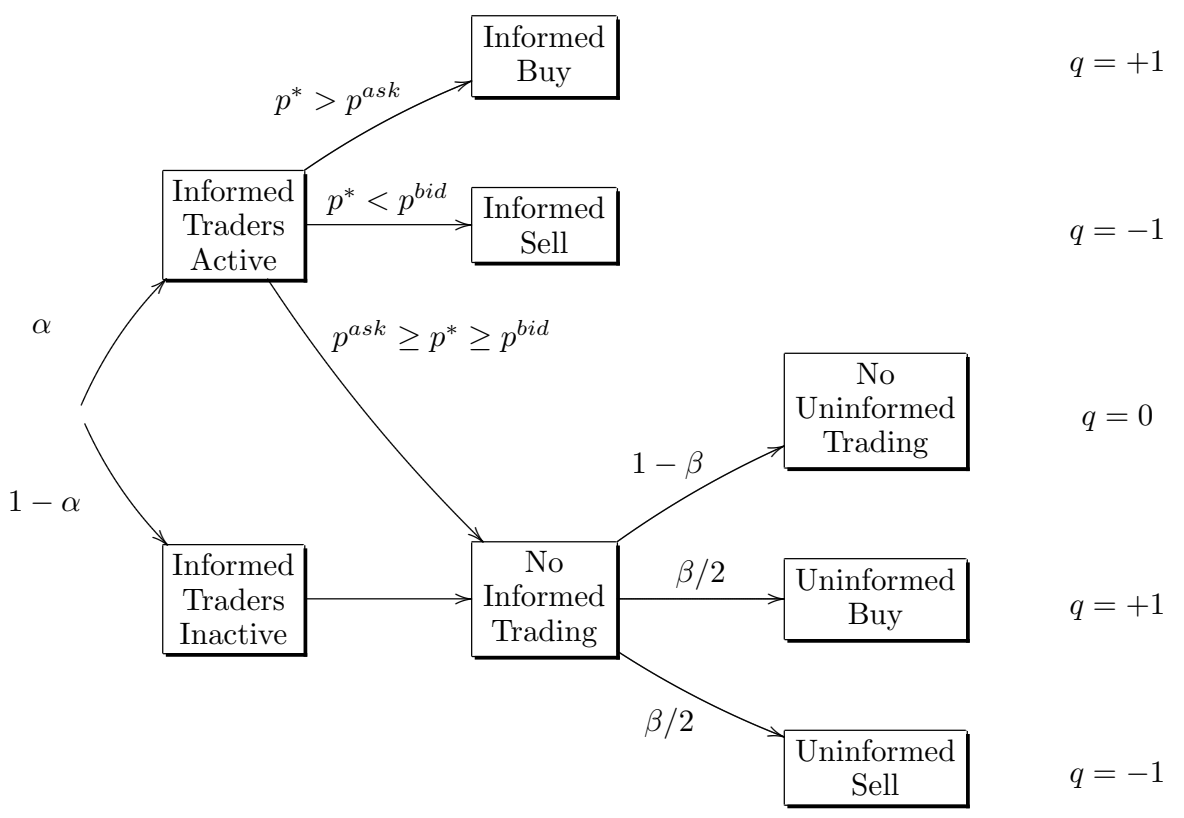

When trading with an informed trader the market maker always loses. His expected loss

\footnotetext{
${ }^{12}$ Easley et al. (2008) estimate time-varying arrival rates of informed and uninformed traders, which they find mutually dependent.
} 
is

$$
L_{n}\left(p_{t}, F(\cdot ; \underline{p}, \bar{p})\right)=-\int_{\underline{p}}^{\bar{p}}\left|\left(p_{t}-p_{t}^{*}\right) E\left(q_{t} \mid p_{t}^{*}, p_{t}, s_{t}\right)\right|^{n} f\left(p_{t}^{*}\right) d p_{t}^{*}
$$

where $E\left(q_{t} \mid p_{t}+s_{t}<p_{t}^{*}\right)=\alpha, E\left(q_{t} \mid p_{t}-s_{t}>p_{t}^{*}\right)=\alpha, E\left(q_{t} \mid p_{t}-s_{t} \leq p_{t}^{*} \leq p_{t}+s_{t}\right)=0$, and $n$ reflects the risk aversion of the market maker. $F(\cdot)$ and $f(\cdot)$ denote the cdf and pdf with

support $[\underline{p}, \bar{p}]$ of the market maker's belief about the latent price. The market maker faces a tradeoff between avoiding losses today and learning quickly. ${ }^{13}$

We add the following assumption, which helps us in greatly simplifying the model without affecting its basic behavior.

Assumption 1 Ex ante $(t=0)$ buys $\left(q_{t}=+1\right)$ and sells $\left(q_{t}=-1\right)$ are equally likely, so that $E\left(q_{t}\right)=0$. There is no "momentum" in uninformed trading, and thus trades are not serially correlated beyond the time of a strong form efficient price change, that is, $E\left(q_{\kappa T+\tau_{1}} \mid q_{\kappa T-\tau_{2}}\right)=0 \quad \forall \kappa, \tau_{1} \in \mathbb{N}_{0}, \forall \tau_{2} \in \mathbb{N}$.

In the following sections 3 and 4 we look at specializations of this general market maker problem and examine the effect of various model setups on the cross-correlation function. For both strong form and semi-strong form efficient returns we first examine the multiperiod case, where private information is not revealed until after many periods. We then specialize to the one-period case, a case where private information becomes public, and worthless, after only one period, where we specifically address the effect of a risk-averse market maker $(n>1)$.

\section{Return/Noise Cross-Correlations in Economic Envi- ronments I: Strong Form Efficient Prices}

Here we characterize cross-correlations in an environment of strong form efficient prices. Accordingly, in this section "efficient price" means "strong form efficient price".

Suppose there is a single change in the strong form efficient price at a known time at which the previous change becomes public knowledge. To fix ideas, let this change occur every $T$ periods. ${ }^{14}$ This allows studying the effect of one efficient price change in isolation.

\footnotetext{
${ }^{13}$ We describe this market maker problem in more detail in Appendix A

${ }^{14}$ The average time $T$ between two changes in the strong form efficient price could be several days, or, more likely, just a few hours. Engle and Patton (2004) and Owens and Steigerwald (2005), for example, find evidence of multiple information arrivals during a calendar day.
} 
We first calculate the correlations between strong form efficient returns

$$
\Delta p_{t}^{*}=\Delta p_{\kappa T}^{*}=\left\{\begin{array}{cc}
\sigma \varepsilon_{\kappa T} & \forall \kappa \in \mathbb{Z} \\
0 & \forall \kappa \notin \mathbb{Z}
\end{array}\right.
$$

and the corresponding noise,

$$
\Delta u_{t}=\Delta p_{t}-\Delta p_{t}^{*}=\Delta p_{t}^{e}+s_{t} q_{t}-s_{t-1} q_{t-1}-\Delta p_{t}^{*}
$$

\subsection{The General Multi-Period Case}

In the period of a change in the strong form efficient price, the expectation about this price changes by ${ }^{15}$

$$
\begin{aligned}
\Delta p_{0}^{e} & =p_{0}^{e}-p_{-1}^{e} \\
& =\sigma \varepsilon_{-T}-\sum_{t=2}^{T}\left(\lambda_{-t} q_{-t}+\omega_{-t}\right)-\omega_{-1}+\omega_{0}
\end{aligned}
$$

and in all other periods by

$$
\Delta p_{t}^{e}=\lambda_{t-1} q_{t-1}+\omega_{t}
$$

From (13) we get for $t=\kappa T$

$$
\Delta u_{0}=\sigma\left(\varepsilon_{-T}-\varepsilon_{0}\right)+s_{0} q_{0}-s_{-1} q_{-1}-\sum_{t=2}^{T}\left(\lambda_{-t} q_{-t}+\omega_{-t}\right)-\omega_{-1}+\omega_{0}
$$

and $\forall t \neq \kappa T$

$$
\Delta u_{t}=\lambda_{t-1} q_{t-1}+s_{t} q_{t}-s_{t-1} q_{t-1}+\omega_{t},
$$

where the first term reflects information-revealing trades, the second and third term reflect the bid-ask bounce, and the last term new non-trade information.

This immediately leads to the contemporaneous cross-covariance

$$
\operatorname{Cov}\left(\Delta p_{t}^{*}, \Delta u_{t}\right)=\frac{\sigma}{T}\left[s_{0} E\left(q_{0} \varepsilon_{0}\right)-\sigma+E\left(\omega_{0} \varepsilon_{0}\right)\right]
$$

For cross-covariance at higher displacements $\tau \in[1 ; T-1]$ we get

$$
\operatorname{Cov}\left(\Delta p_{t-\tau}^{*}, \Delta u_{t}\right)=\frac{\sigma}{T}\left[\left(\lambda_{\tau-1}-s_{\tau-1}\right) E\left(q_{\tau-1} \varepsilon_{0}\right)+s_{\tau} E\left(q_{\tau} \varepsilon_{0}\right)+E\left(\omega_{\tau} \varepsilon_{0}\right)\right]
$$

\footnotetext{
${ }^{15}$ As a shorthand notation we use $p_{x} \equiv p_{\kappa T+x} \forall \kappa, x \in \mathbb{Z}$.
} 
for cross-covariance at displacement $T$

$$
\operatorname{Cov}\left(\Delta p_{t-T}^{*}, \Delta u_{t}\right)=\frac{\sigma}{T}\left[\sigma-s_{T-1} E\left(q_{T-1} \varepsilon_{0}\right)-\sum_{i=0}^{T-2} \lambda_{i} E\left(q_{i} \varepsilon_{0}\right)-\sum_{i=0}^{T-1} E\left(\omega_{i} \varepsilon_{0}\right)\right],
$$

and for all higher order displacements $\tau>T$

$$
\operatorname{Cov}\left(\Delta p_{t-\tau}^{*}, \Delta u_{t}\right)=0 .
$$

Now consider $\operatorname{Var}\left(\Delta p_{t}^{*}\right)$ and $\operatorname{Var}\left(\Delta u_{t}\right)$. The strong form efficient price has unconditional variance

$$
\operatorname{Var}\left(\Delta p_{t}^{*}\right)=\frac{1}{T} \operatorname{Var}\left(\sigma \varepsilon_{0}\right)=\frac{\sigma^{2}}{T},
$$

and the corresponding noise has unconditional variance

$$
\begin{aligned}
\operatorname{Var}\left(\Delta u_{t}\right) & =\frac{1}{T} \sum_{i=0}^{T-1} \operatorname{Var}\left(\Delta u_{i}\right) \\
& =\frac{1}{T}\left\{2 \sigma^{2}+\sum_{i=0}^{T-1}\left(\phi_{i} s_{i}^{2}+\phi_{i-1} s_{i-1}^{2}\right)-2 \sigma s_{T-1} E\left(q_{T-1} \varepsilon_{0}\right)-2 \sigma \sum_{i=0}^{T-2} \lambda_{i} E\left(q_{i} \varepsilon_{0}\right)\right. \\
& -2 \sigma s_{0} E\left(q_{0} \varepsilon_{0}\right)+2 s_{T-1} \sum_{i=0}^{T-2} \lambda_{i} E\left(q_{i} q_{T-1}\right)+E\left[\left(\sum_{i=0}^{T-2} \lambda_{i} q_{i}\right)^{2}\right]+E\left(\omega_{0}^{2}\right) \\
& +E\left[\sum_{i=0}^{T-1} \omega_{i}^{2}\right]+2 s_{0} E\left(q_{0} \omega_{0}\right)-2 \sigma \sum_{i=0}^{T} E\left(\varepsilon_{i} \omega_{i}\right)+2 s_{T-1} \sum_{i=0}^{T-1} E\left(q_{T-1} \omega_{i}\right) \\
& +\sum_{i=1}^{T-1}\left(\phi_{i-1} \lambda_{i-1}\left(\lambda_{i-1}-2 s_{i-1}\right)+2\left(\lambda_{i-1}-s_{i-1}\right)\left(s_{i} E\left(q_{i-1} q_{i}\right)+E\left(q_{i-1} \omega_{i}\right)\right)\right. \\
& \left.\left.+E\left(\omega_{i}^{2}\right)+2 s_{i} E\left(q_{i} \omega_{i}\right)\right)\right\},
\end{aligned}
$$

where

$$
\phi_{t}=E\left(q^{2}\right)=E[\operatorname{Prob}(q=+1 \vee q=-1)]=\beta+(1-\beta) \alpha\left[1-F\left(p_{t}^{e}+s_{t}\right)+F\left(p_{t}^{e}-s_{t}\right)\right] .
$$

Using (16) the contemporaneous cross-correlation is

$$
\operatorname{Corr}\left(\Delta p_{t}^{*}, \Delta u_{t}\right)=\frac{s_{0} E\left(q_{0} \varepsilon_{0}\right)-\sigma+E\left(\omega_{0} \varepsilon_{0}\right)}{\sqrt{T \operatorname{Var}\left(\Delta u_{t}\right)}} .
$$

All other cross-correlations can be obtained analogously using (17) to (19). 
The term $E\left(q_{\tau} \varepsilon_{0}\right)$ enters the expressions for the cross-covariance (16)-18 linearly and enters the denominator of the cross-correlation under a square root. Because this term decreases the share of uninformed trades, the contemporaneous cross-correlation is the smaller, the less informed traders are active. Only in absence of any informed traders we have $E\left(q_{\tau} \varepsilon_{0}\right)=0$, and the market microstructure reduces to a bid-ask bounce, as in Roll (1984). Even in this case, shown in the first row of Table 1, the latent price and noise are not independent. The contemporaneous cross-correlation $(16)$ is negative, the cross-correlations at displacement $T$ is positive and all other cross-correlations are zero.

Because of order splitting, effective spreads have become very small for liquid assets. If the spread sufficiently small, ${ }^{16}$ the contemporaneous cross-correlation is negative, because in this case $p_{t}$ shows barely any instantaneous reaction to $\Delta p_{t}^{*} \cdot{ }^{17}$ Likewise, if the spread roughly matches the adverse selection coefficient, by (17) the cross-correlations at displacements one up to $T-1$ are positive, which reflects that the more the market maker learns, the closer $p_{t}$ gets to $p_{t}^{*}$, and the more noise shrinks to zero. If, additionally, the adverse selection coefficient $\lambda$ and extra information $\omega$ in all periods are sufficiently small, by (18) the cross-correlation at displacement $T$ is positive.

In general, however, the sign of the cross-correlations depends on the behavior of the market maker and traders. We now turn to models that allow us to introduce these explicitly.

\subsection{Special Multi-Period Cases}

Because the market maker loses in every trade with an informed trader, he has an incentive to find out the strong form efficient price. He learns about the informed traders' private information by setting prices and observing the resulting trades. As he learns over time "by experimentation", ${ }^{18}$ the value of private information of the informed trader slowly vanishes. Although there are many possible interactions of strategic actions by market participants, we will see that rational behavior ensures that they all share the same cross-correlation sign pattern and differ only in the absolute value of the cross-correlation.

The market maker does not observe $p_{t}^{*}$ directly, but only signals which allow him to narrow down the range of the current $p_{t}^{*}$ level. He observes in particular the response of traders to his previous price quote and uses this signal to revise his quote. Because the strong form efficient price, $p_{t}^{*}$, by assumption 12 does not change after the initial jump for $T$ periods, the market maker can use the entire sequence of signals to learn $p_{t}^{*}$ over time. His

\footnotetext{
${ }^{16} \mathrm{~A}$ sequence of only bid prices (or only ask prices) is equivalent to $s_{t}=0 \quad \forall t$.

${ }^{17} \rho_{0}$ is negative, but strictly larger than negative one. This obtains, because $p_{t}$ responds every period to noisy signals about $p_{0}^{*}$, which increases the noise variance.

${ }^{18}$ Aghion et al. (1991), Aghion et al. (1993)
} 
optimization task is to quote prices that minimize his losses by learning about $p_{t}^{*}$ as quickly as possible.

The recursive problem of the market maker is hard to solve, and in particular there are in general no closed form policy functions $p_{t}^{b i d}$ and $p_{t}^{a s k}$. Therefore we follow the market microstructure literature by discussing interesting polar cases, which can be solved because $f\left(p_{t}^{*}\right)$ is degenerate. ${ }^{19}$

\subsubsection{No Strategic Traders}

Consider a market in which the market maker observes only a noisy signal of whether $p_{t}^{*}$ has changed, but in which traders do not behave strategically yet. The market maker has to learn both about the quality of the signal and about the latent price. Glosten and Milgrom (1985) describe a market maker who - as in our general setup - does not know whether he is trading with an informed or an uninformed trader and thus cannot tell whether the direction of trade, $q_{t}$, contains information. For example, the market maker cannot tell whether a "buy" originates from an informed trader, in which case it would indicate an increase in the strong form efficient price, or whether it is just a random trade of an uninformed trader. Thus, a noisy "buy" increases the likelihood of an increase in the strong form efficient price less than a "buy" in a "perfect signal" environment.

As useful illustration is the stylized model of Easley and O'Hara (1992), which allows us to derive the cross-correlation pattern explicitly. As in our general setup in section 2.3 informed traders are active with probability $\alpha$. The two possible latent price levels, $\underline{p}^{*}$ and $\bar{p}^{*}>\underline{p}^{*}{ }^{20}$ and the probability $\gamma$ of a low latent price are publicly known, but the actual realization of $p_{t}^{*}$ is not.

The direction-of-trade signal, $q_{t}$, is thereby uncertain in two ways. ${ }^{21}$ Not only does the market maker not know if a specific trade originates from informed traders, thereby being informative; the market maker does not even know if there are any informed traders. He learns by updating in a Bayesian manner his belief about the probabilities that nobody observed a signal, that informed traders observed a high $p_{t}^{*}$, or that they observed a low $p_{t}^{*}$, using his information set of all previous quotes and trades, $\Omega_{t}$. Even non-trading intervals contain information about $p_{t}^{*}$, because they lower the probability that informed traders are

\footnotetext{
${ }^{19}$ See Appendix A.2. We assume in the following section 3.2.1 that market makers are risk neutral $(n=1)$ and limit our discussion to the mid price under a constant spread in order to study the learning effect in isolation.

${ }^{20}$ The strong form efficient price is not a martingale here.

${ }^{21}$ The case of signal certainty, which implies the absence of any uninformed traders, is trivial here: Because $p_{t}^{*}$ can assume only one of two price levels, the first trade reveals the true strong form efficient price. Until the first trade occurs, the expected efficient price is $\gamma \underline{p}^{*}+(1-\gamma) \bar{p}^{*}$.
} 
active. $^{22}$

Denote $\beta_{\tau,\left\{\bar{p}^{*}\right\}}$ the belief at time $t+\tau$ that a high latent price has been observed, $\beta_{\tau,\left\{\underline{p}^{*}\right\}}$ the belief that a low latent price has been observed and $\beta_{\tau,\{\}}$ the belief that nobody has observed any signal, all conditional on $\Omega_{t} \cup\left\{q_{t}\right\}$. The market maker sets under perfect competition

$$
\begin{aligned}
p_{\tau}^{b i d}-\underline{p}^{*} & =\beta_{\tau,\left\{\underline{p}^{*}\right\}}\left(1-\beta_{\tau,\{\}}\right) \underline{p}^{*}+\beta_{\tau,\left\{\bar{p}^{*}\right\}}\left(1-\beta_{\tau,\{\}}\right) \bar{p}^{*}+\beta_{\tau,\{\}} \frac{\underline{p}^{*}+\bar{p}^{*}}{2}-\underline{p}^{*} \\
& =\left(\beta_{\tau,\left\{\bar{p}^{*}\right\}}+\frac{\beta_{\tau,\{\}}}{2}\right)\left(\bar{p}^{*}-\underline{p}^{*}\right) .
\end{aligned}
$$

A sufficiently large $\tau$ allows invoking a law of large numbers for the observations included in the market maker believes. Easley and O'Hara (1992) show for the case that traders observe a low latent price that $\beta_{\tau,\left\{\bar{p}^{*}\right\}}=\exp \left(-r_{1} \tau\right)$ and $\beta_{\tau,\{\}}=\exp \left(-r_{2} \tau\right)$ for some $r_{1}, r_{2}>0$. Hence for large $\tau$ the bid price $p_{t}^{\text {bid }}$ converges to $\underline{p}^{*}$ almost surely at the exponential learning rate $r=\min \left(r_{1}, r_{2}\right)$ in clock time.

$$
p_{t}^{\text {bid }} \stackrel{\text { a.s. }}{\rightarrow} \underline{p}^{*}
$$

An analogous result applies to the convergence of the ask price to $\underline{p}^{*}$. Thus transaction prices converge to the strong form efficient price in clock time at exponential rates for large $\tau .^{23}$

The following proposition summarizes the cross-correlations in Easley and O'Hara (1992)type models. It considers only the dominant exponential learning pattern, and ignores all terms which disappear at a faster rate as $\tau$ gets large.

\section{Proposition 1 (Cross-correlations in the Easley-O'Hara model)}

The contemporaneous cross-correlation in the Easley and O'Hara (1992) model is

$$
\operatorname{Corr}\left(\Delta p_{t}^{*}, \Delta u_{t}\right)=-\frac{1+e^{-r(T-1)}}{2 \sqrt{K}}<0
$$

and the cross-correlations at sufficiently large nonzero displacements follow

$$
\begin{gathered}
\operatorname{Corr}\left(\Delta p_{t-\tau}^{*}, \Delta u_{t}\right)=\frac{e^{r}-1}{2 \sqrt{K}} e^{-r \tau}>0, \forall \tau \in[1, T-1] \\
\operatorname{Corr}\left(\Delta p_{t-T}^{*}, \Delta u_{t}\right)=\frac{e^{-r(T-1)}}{2 \sqrt{K}}>0
\end{gathered}
$$

\footnotetext{
${ }^{22} \mathrm{~A}$ variation of this setup is the model of Diamond and Verrecchia (1987), where short selling constraints cause periods of no trading to be a noisy signal of a low latent price.

${ }^{23}$ This corresponds to proposition 6 in Easley and O'Hara (1992). The result has been derived for calendar time sampling. Tick time sampling leads to lower convergence rates, because it misses the no-trade periods, which reveal information as well: During trading days in which no trader has observed the latent price there are more no-trade periods than during trading days in which some have.
} 
where $K=K(r, T)$.

Proof: See Appendix B.1.

As before, the contemporaneous correlation is negative, and approaches its minimum for small $r$ and small $T$. Furthermore, for $\tau \in[1, T-1]$,

$$
\operatorname{Corr}\left(\Delta p_{t-\tau}^{*}, \Delta u_{t}\right)=\left(\frac{1}{e^{r}}\right)^{\tau-1} \operatorname{Corr}\left(\Delta p_{t-1}^{*}, \Delta u_{t}\right)
$$

That is, the cross-correlation of the strong form efficient price decays geometrically to zero until $\tau=T$.

Figure 4: Cross-Correlation Functions $\rho_{\tau}$ of the Strong Form Efficient Price

(a) Noisy Signal $(r=0.5, T=5)$

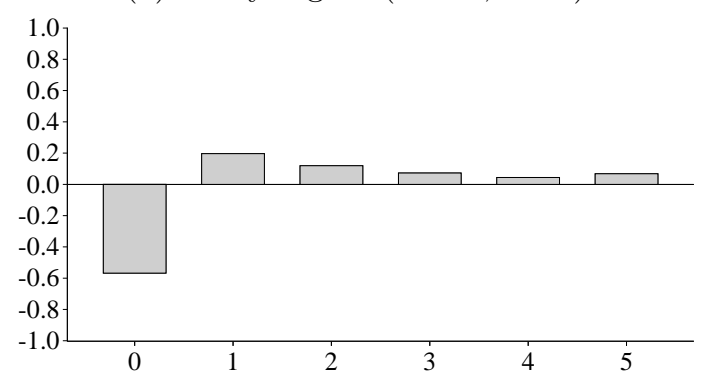

(c) Strategic Traders $(T=5)$

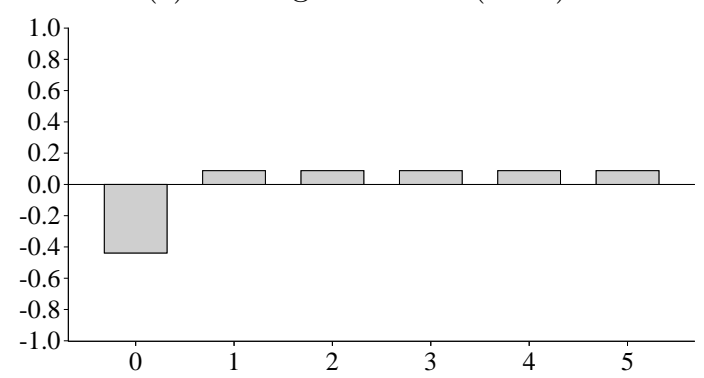

(e) Low Risk Aversion ( $T=1)$

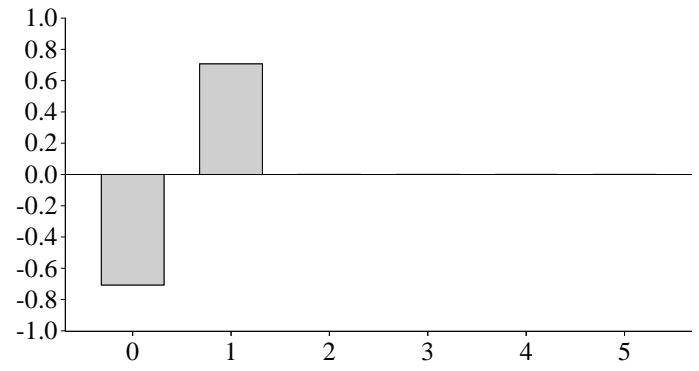

(b) Noisy Signal $(r=2, T=5)$

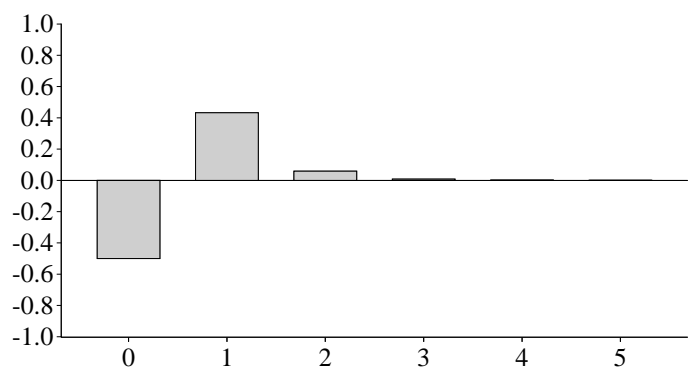

(d) Strategic Traders $(T=2)$

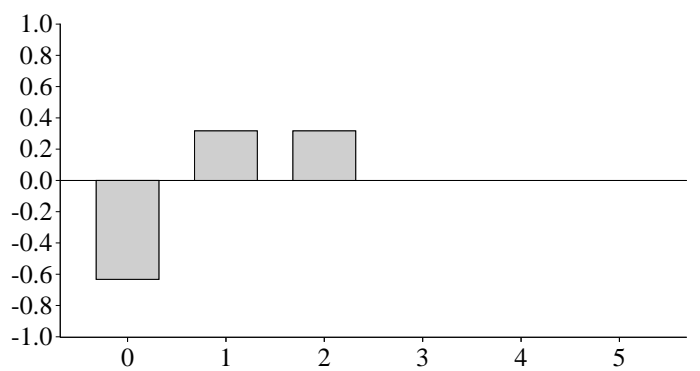

(f) High Risk Aversion ( $T=1)$

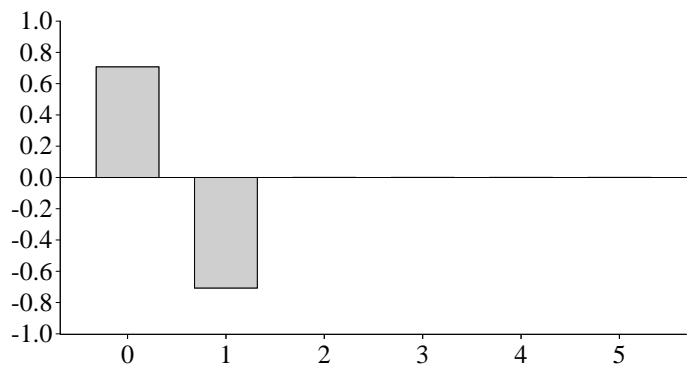

In the first row of Figure 4 we graph this cross-correlation function. We show the cross- 
correlation pattern for an Easley-O'Hara (1992)-type model for a learning rate of $r=0.5$ in the upper left panel, and for a faster learning rate of $r=2$ in the upper right panel.

We summarize the results for the Easley-O'Hara (1992) model in the third row of Table 1. This decay pattern is not unique to the Easley-O'Hara (1992) model. In Appendix C we show that this cross-correlation pattern holds under signal certainty as well. In this case it is optimal for market markers to learn the latent price by repeated bisections. Often, optimal learning stops before $p^{*}$ is reached (Aghion et al., 1991), e.g. if the spread is large or if risk aversion is small. In that case the cross-correlations cut off at some $\tau<T$.

More generally, Glosten and Milgrom (1985) show that if learning is costless, the expectations of market makers and traders necessarily converge as the number of trades increases. We summarize these qualitative results in the second row of Table 1. Because of the uncertainty of whether a trade reflects information or just noise, the market maker faced with a noisy signal adjusts only partially. Therefore, whereas the cross-correlations under a noisy signal have the same signs as under signal certainty, their absolute values are all dampened toward zero.

Table 1: Strong Form Efficient Cross-Correlations in Multi-period Models

\begin{tabular}{|c|c|c|c|c|c|c|}
\hline $\begin{array}{c}p_{t}^{*} \text { mar- } \\
\text { tingale }\end{array}$ & signal & $\begin{array}{l}\text { traders } \\
\text { strategic }\end{array}$ & $\rho_{0}$ & $\begin{array}{c}\rho_{\tau} \\
\tau \in[1, T-1]\end{array}$ & $\rho_{T}$ & $\begin{array}{c}\rho_{\tau} \\
\tau>T\end{array}$ \\
\hline yes & none & n.a. & $\rho_{0}<0$ & 0 & $-\rho_{0}$ & 0 \\
\hline yes & $\begin{array}{c}\text { certain/ } \\
\text { noisy }\end{array}$ & no & $\rho_{0}<0$ & $\rho_{\tau-1}>\rho_{\tau}>0$ & $\rho_{T}>0$ & 0 \\
\hline no & noisy & no & $-\frac{1+e^{-r(T-1)}}{2 \sqrt{K(r, T)}}$ & $\frac{-e^{-r \tau}+e^{-r(\tau-1)}}{2 \sqrt{K(r, T)}}$ & $\frac{e^{-r(T-1)}}{2 \sqrt{K(r, T)}}$ & 0 \\
\hline yes & noisy & yes & $-\sqrt{\frac{T}{T^{2}+1}}$ & $\sqrt{\frac{1}{T\left(T^{2}+1\right)}}$ & $\sqrt{\frac{1}{T\left(T^{2}+1\right)}}$ & 0 \\
\hline
\end{tabular}

Notes: We show $\rho_{\tau}=\operatorname{Corr}\left(\Delta p_{t-\tau}^{*}, \Delta u_{t}\right)$ in multiperiod models $(T>1)$ under risk neutrality $(n=1)$.

\subsubsection{Strategic Traders}

Because the market maker cannot distinguish informed trades from uninformed ones, informed traders can act strategically. The aim of strategic behavior of informed traders is to make the signals about $p_{t}^{*}$ conveyed by their orders as noisy as possible, while still executing the desired trades. By mimicing uninformed traders they keep the market maker unaware about the change in $p_{t}^{*}$. Because the market maker observes the order flow and uses it to detect informed trading, the informed traders stretch their orders over a long time period such that detecting an abnormal trading pattern becomes difficult. The market maker will, 
of course, notice the imbalance in trades over time. By sequentially updating his belief about $p_{t}^{*}$ based on the history of trades he still learns about $p_{t}^{*}$, but, because of the strategic behavior of traders, at the slowest possible rate.

Markets of this type have been described in Kyle (1985) and Easley and O'Hara (1987). In the following we discuss the cross-correlation function implied by the Kyle (1985) model. The strategic behavior described by Kyle (1985) requires that exactly one trader is informed, or that all informed traders build a monopoly and coordinate trading. Here, the market maker does not maximize a particular objective function, he merely ensures market efficiency, i.e. sets the market price such that it equals the expected strong form efficient price, $p_{t}^{e}$, given the observed aggregate trading volume from informed and uninformed traders. The only optimizer in this model is the (risk neutral) informed trader who optimally spreads his orders over the day to minimize the (unfavorable) price reaction of the market maker. Thereby he maximizes his expected total daily profit using his private information and taking the price setting rule $\Delta p_{t}\left(\Omega_{t}\right)$ of the market makers as given. Effectively, the informed trader trades most when the sensitivity of prices to trading quantity is small.

Kyle (1985) assumes a linear reaction function of the market maker, which implies $\lambda_{t}=\lambda$ $\forall t \in[1, T]$, and a linear reaction function for the informed trader, which implies $q_{t}=q$ $\forall t \in[0, T-1]$. Under these assumptions he shows that in expectation the market price approaches the latent price linearly, not exponentially. The reason for this difference is that the market maker in Easley and O'Hara (1992) updates his beliefs in a Bayesian manner, whereas in Kyle (1985) the market maker's actions are constrained to market clearing. The other key feature of this model is that by the end of the trading day - just before $p_{t}^{*}$ would be revealed - the market price reflects all information.

From the continuous auction equilibrium in Kyle (1985) the price change at time $t$ is

$$
d p^{e}(t)=\frac{p^{*}-p^{e}(t)}{T-t} d t+\sigma d z, t \in[0, T]
$$

The innovation term $d z$ is white noise with $d z \sim N(0,1)$ and reflects the price impact of uninformed traders. This stochastic differential equation has the solution

$$
p^{e}(t)=\frac{t}{T} p^{*}+\frac{T-t}{T} p^{e}(0)+(T-t) \int_{0}^{t} \frac{\sigma}{T-s} d B_{s}
$$

where $d B_{s} \equiv d z .^{24}$ The increments of the expected price over a discrete interval of time

\footnotetext{
${ }^{24}$ The third term reflects uninformed trading. It has an expected value of zero, and the impact of this random component increases during the early trading day and decreases lateron - its contribution to $p^{e}(t)$ is therefore hump-shaped over time.
} 
follow therefore

$$
\Delta p_{\tau}^{e}=\frac{\Delta p_{0}^{*}}{T}+(T-\tau) \int_{\tau-1}^{\tau} \frac{\sigma}{T-s} d B_{s}-\int_{0}^{\tau-1} \frac{\sigma}{T-s} d B_{s}
$$

The following proposition presents the cross-correlations for the Kyle (1985) model.

\section{Proposition 2 (Cross-correlations in the Kyle model)}

The contemporaneous cross-correlation in Kyle (1985) is

$$
\operatorname{Corr}\left(\Delta p_{t}^{*}, \Delta u_{t}\right)=-\sqrt{\frac{T}{T^{2}+1}}
$$

the cross-correlations at displacements $\tau \in[1 ; T]$ are

$$
\operatorname{Corr}\left(\Delta p_{t-\tau}^{*}, \Delta u_{t}\right)=\sqrt{\frac{1}{T\left(T^{2}+1\right)}}
$$

and all higher order cross-correlations are zero.

Proof: See Appendix B.2.

The cross-covariance at nonzero displacements is positive because of market maker learning. It is constant because of the strategic behavior of traders, which spread new information equally over time. This maximizes the time it takes the market maker to include the entire strong form efficient price change in his quotes. The more periods, the more pronounced is the negative contemporaneous cross-correlation, and the smaller are the cross-correlations at nonzero displacements.

We plot the cross-correlation function given by Proposition 2 in the second row of Figure 4. We show the cross-correlation function a Kyle (1985)-type model under modestly frequent changes in the latent price $(T=5)$ in the left panel, and for more frequent changes $(T=2)$ in the right panel. Table 1 compares standard multiperiod market microstructure models. In contrast to markets with nonstrategic traders, which display decaying lagged crosscorrelations (row 3), markets with strategic traders display constant lagged cross-correlations (row 4).

\subsection{One-Period Case}

In this section we consider the extreme case of markets in which $p_{t}^{*}$ automatically becomes public information at the end of each period, i.e. $\omega_{t}=p_{t-1}^{*}-\tilde{p}_{t-1}^{e}$ and $T=1$. This allows us to investigate the impact of risk aversion for the cross-correlation pattern. $p_{t-1}^{*}$ 
is thus known when the market maker decides on $p_{t}$, and by (1) and (4) $p_{t}^{e}=p_{t}^{*}-\sigma \varepsilon_{t}$. The free distribution of information removes any incentive for informed traders to behave strategically. They therefore react immediately, which implies $E\left(q_{t-\tau} \varepsilon_{t}\right)=0 \quad \forall \tau \neq 0$ and trades are serially uncorrelated, i.e. $E\left(q_{t} \mid q_{t-1}\right)=0$. For the market maker all periods are identical, and therefore the spread and reaction parameters are both constant over time, i.e. $s_{t}=s$ and $\lambda_{t}=\lambda \forall t$.

Because $T=1$ the market maker's recursive problem ${ }^{25}$ collapses to a sequence of single period problems. This by itself pins down the shape of the cross-correlation function. By (16) and (18) the cross-correlation at displacement one has the opposite sign and same absolute value as the contemporaneous cross-correlation, and by (19) all cross-correlations at displacements larger than one are zero.

In order to pin down the contemporaneous cross-correlation, we now turn to specific models.

\subsubsection{No Market Maker Information}

We start with our baseline assumption that the market maker at time $t$ has no information whatsoever about $\Delta p_{t}^{*}$. Plugging $T=1, s_{t}=s$, and $\lambda_{t}=\lambda$, and thus $\phi_{t}=\phi$, into the general multiperiod results derived in section 3.1 gives

Proposition 3 (Strong form cross-correlation, one period model)

$$
\begin{gathered}
\operatorname{Corr}\left(\Delta p_{t}^{*}, \Delta u_{t}\right)=\frac{1}{\sqrt{2}} \frac{s E\left(q_{t} \varepsilon_{t}\right)-\sigma}{\sqrt{\phi s^{2}+\sigma^{2}-2 s \sigma E\left(q_{t} \varepsilon_{t}\right)}} \\
\operatorname{Corr}\left(\Delta p_{t-1}^{*}, \Delta u_{t}\right)=-\operatorname{Corr}\left(\Delta p_{t}^{*}, \Delta u_{t}\right)
\end{gathered}
$$

Proof: See Appendix B.3.

As the following Proposition 4 shows, if there is trading in every period $(\beta=1)$, and thus $\phi=1$ ), then the cross-correlation (24) can be bounded from above and below.

\section{Proposition 4 (Bounds of contemporaneous cross-correlation)}

$$
-\frac{1}{\sqrt{2}} \leq \operatorname{Corr}\left(\Delta p_{t}^{*}, \Delta u_{t}\right) \leq 0
$$

\footnotetext{
${ }^{25}$ See Appendix A.2.
} 
Proof: See Appendix B.4.

Note that the cross-correlation reaches the lower bound for zero spread. Thus for mid prices, or extremely small spreads due to order splitting, the cross-correlation is highest. For transaction prices the contemporaneous cross-correlation is less pronounced. The contemporaneous cross-correlation for mid prices is negative, because $p_{t}^{\text {mid }}$ does not react at all to the change in the strong form efficient price in the same period. ${ }^{26}$ It differs from negative unity because market prices move in adjustment to the strong form efficient return one period earlier.

We summarize these results in the upper two rows of Table 2, Compared to the multiperiod case $(T>1)$ the absolute value of the cross-correlation at lag one is large, because all information is revealed. Cross-correlations at any displacement beyond one, in contrast, are all zero.

Table 2: Cross-Correlations in One-period Models

\begin{tabular}{ccc|ccc}
\hline $\begin{array}{c}\text { efficient } \\
\text { price }\end{array}$ & spread & $\begin{array}{c}\text { loss } \\
\text { function }\end{array}$ & $\rho_{0}$ & $\rho_{1}$ & $\begin{array}{c}\rho_{\tau} \\
\tau>1\end{array}$ \\
\hline strong & 0 & any & $-\frac{1}{\sqrt{2}}$ & $\frac{1}{\sqrt{2}}$ & 0 \\
strong & $\geq 0$ & $\begin{array}{c}\text { any } \\
\text { high } n+\end{array}$ & $-\frac{1}{\sqrt{2}} \leq \rho_{0}<0$ & $-\rho_{0}$ & 0 \\
strong & $\geq 0$ & $\rho_{0}>0$ & $-\rho_{0}$ & 0 \\
\hline semi-strong & $\geq 0$ & quadratic & $-\frac{1}{\sqrt{2}} \leq \rho_{0} \leq \frac{1}{\sqrt{2}}$ & $-\rho_{0}$ & 0 \\
semi-strong & $\in[0, \lambda[$ & any & ambiguous & $\rho_{1}>0$ & 0 \\
semi-strong & $\lambda$ & any & 0 & 0 & 0 \\
semi-strong & $\geq \lambda$ & any & ambiguous & $\rho_{1}<0$ & 0 \\
\hline
\end{tabular}

Notes: The upper half of the table reports $\rho_{\tau}=\operatorname{Corr}\left(\Delta p_{t-\tau}^{*}, \Delta u_{t}\right)$ under no extra market maker information in rows 1 and 2, and under extra market maker information $\left\{\operatorname{sgn}\left(\varepsilon_{t}\right)\right\}$ in row 3 . The lower half reports $\rho_{\tau}=\operatorname{Corr}\left(\Delta \tilde{p}_{t-\tau}, \Delta u_{t}\right)$.

\subsubsection{Incomplete Market Maker Information and Risk Aversion}

Previously our market maker set prices knowing $p_{t-1}^{*}$ but without information about the strong form efficient return in period $t$. Now we show that under risk aversion the existence of extra information about the direction of the change in the latent price, i.e. $\left\{\operatorname{sgn}\left(\varepsilon_{t}\right)\right\}$,

\footnotetext{
${ }^{26}$ This is an instance of the price stickiness that Bandi and Russell (2006) show to generate "mechanically" a negative contemporaneous cross-correlation.
} 
can invert the cross-correlation pattern. Observing $\left\{\operatorname{sgn}\left(\varepsilon_{t}\right)\right\}$ before setting his price $p_{t}^{\text {mid }} \equiv$ $p_{t}^{e}$ allows the market maker to adjust the mid price before any informed trader can take advantage of the latent price change. The market maker updates his prior belief about $p_{t}^{*}$, summarized by the distribution $p_{t}^{*} \sim f\left(p_{t-1}^{*}, \sigma^{2}\right)$, with the signal $\left\{\operatorname{sgn}\left(\varepsilon_{t}\right)\right\}$. For convenience of exposition we use

Assumption 2 The probability density function of $\varepsilon_{t}$ is symmetric around its zero mean, monotonically increasing on $]-\infty ; 0]$ and monotonically decreasing on $[0 ; \infty[$.

The updated distribution $\tilde{f}(\cdot)$ differs from $f(\cdot)$ in that it is truncated from below or above at $p_{t}^{*}=p_{t-1}^{*}$ when $\operatorname{sgn}\left(\varepsilon_{t}\right)>0$ or $\operatorname{sgn}\left(\varepsilon_{t}\right)<0$, respectively. The left panels of Figure 5 illustrate the posterior distribution after observing the signal $\left\{\operatorname{sgn}\left(\varepsilon_{t}\right)=+1\right\}$ and $\left\{p_{t-1}^{*}=0\right\}$ : If the prior is a normal distribution, the posterior is given by the half normal in the upper left panel. If the prior is a tent distribution, the posterior is given by the triangular distribution in the lower left panel.

Figure 5: Optimal Predictor $p(n)$ under Half-Normal and Triangular Distributions

(a) Half-Normal Distribution

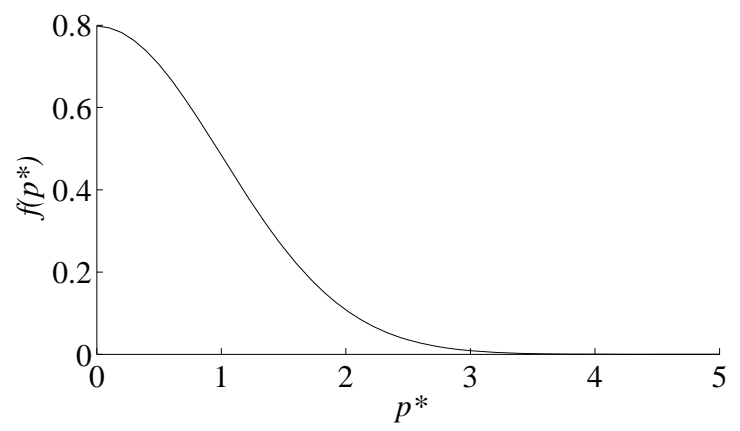

(c) Triangular Distribution

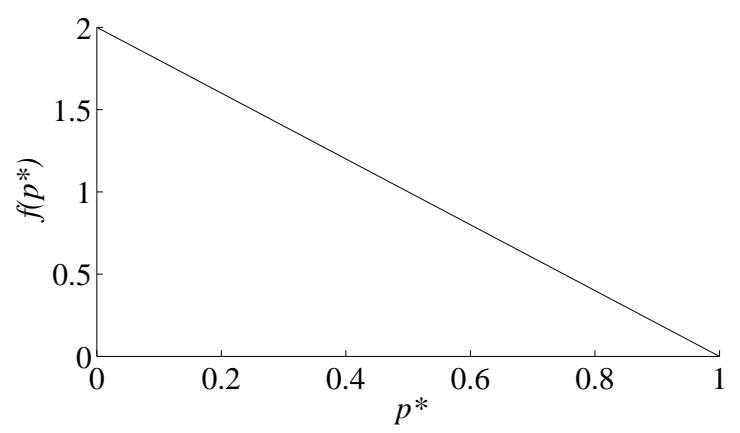

(b) Optimal Predictor for Half-Normal

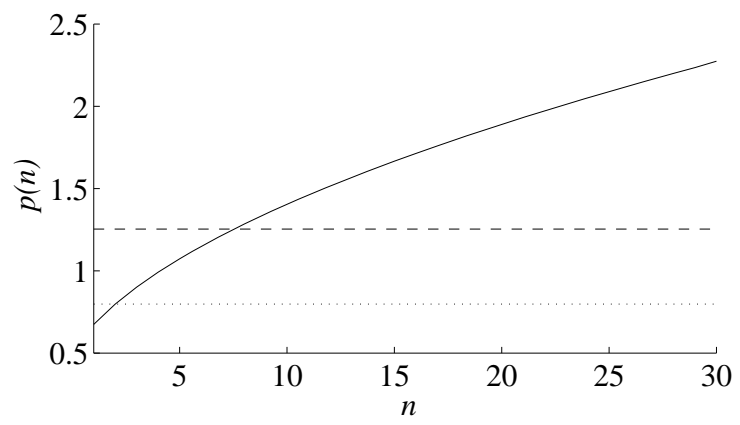

(d) Optimal Predictor for Triangular

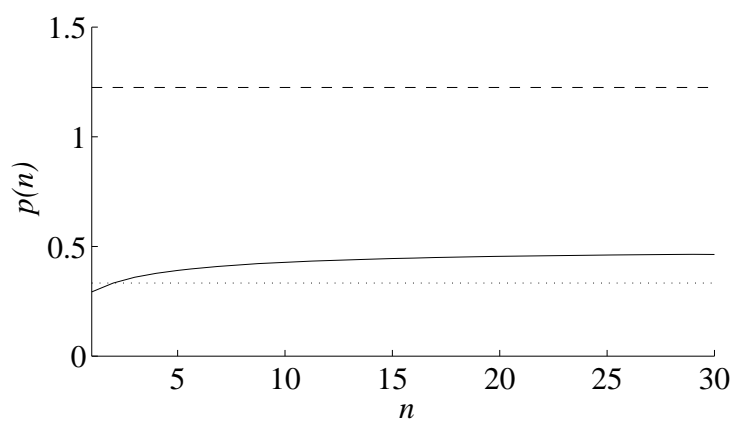

Notes: The left panels are two examples of market maker believes about the strong form efficient price after observing $\left\{\operatorname{sgn}\left(\varepsilon_{t}\right)=+1\right\}$. The right panels show the corresponding optimal predictors, $p^{\text {mid }}(n)$, as a function of risk aversion $n$. The dotted line marks $E\left(\Delta p_{t}^{*}\right)$, and the dashed line marks $\frac{\sigma}{E\left(\left|\Delta p_{t}^{*}\right|\right)}=\frac{\sqrt{E\left(\varepsilon^{2}\right)}}{E(|\varepsilon|)}$. 
After observing this signal and $p_{t-1}^{*}$, the market maker quotes a bid and an ask price for the following period, taking the spread $s$ as given:

$$
p_{t}=p_{t-1}^{*}+s q_{t}+R\left(\left\{\operatorname{sgn}\left(\varepsilon_{t}\right)\right\}\right)
$$

This equation resembles (5), augmented by the market maker response function $R(\cdot)$ to the extra information. ${ }^{27}$ The function $R(\cdot)$ depends in particular on the market maker's risk aversion, $n$.

An approximation ${ }^{28}$ to the problem of choosing $p_{t}^{\text {mid }}(n)$ based on loss function $(11)$ is

$$
p^{\text {mid }}(n)=\underset{x \in\left[\underline{p}^{*}, \bar{p}^{*}\right]}{\operatorname{argmax}}-\int_{\underline{p}^{*}}^{x}\left(x-p^{*}\right)^{n} f\left(p^{*}\right) d p^{*}-\int_{x}^{\bar{p}^{*}}\left(p^{*}-x\right)^{n} f\left(p^{*}\right) d p^{*} .
$$

For some values of $n$, explicit solutions to 26 are available, which we list in the following proposition.

\section{Proposition 5 (Optimal mid price)}

$$
\begin{aligned}
p^{\text {mid }}(1) & =\operatorname{Median}\left(p_{t}^{*}\right) \\
p^{\text {mid }}(2) & =\mathrm{E}\left(p_{t}^{*}\right) \\
p^{\text {mid }}(\infty) & =\operatorname{Midsupport}\left(p_{t}^{*}\right) .
\end{aligned}
$$

Proof: See Appendix B.5.

The higher the risk aversion $n$, the more sensitive is the expected loss, $L_{n}\left(p_{t}, F\left(\cdot, \underline{p}^{*}, \bar{p}^{*}\right)\right)$, to the support of $p_{t}^{*}$, that is, to $\underline{p}^{*}$ and $\bar{p}^{*}$. A well-known result is that the optimal choice for a risk neutral market maker $(n=1)$ is to set $p_{t}^{\text {mid }}$ equal to the median of $f(\cdot)$, and for a modestly risk averse market maker $(n=2)$ to the mean. An extremely risk averse $(n \rightarrow \infty)$ market maker follows the most robust pricing role possible: He minimizes his expected loss at the price in the middle of the support of $f(\cdot)$, i.e. $p_{t}=\frac{p^{*}+\bar{p}^{*}}{2}$.

The right panels of Figure 5 illustrate this. They plot the market price as a function of risk aversion $n$ and show that as risk aversion $n$ grows, $p^{\text {mid }}(n)$ moves monotonically from the median of $f(\cdot)$ to the midpoint of the support of $f(\cdot) \cdot{ }^{29}$ The upper right panel shows this for

${ }^{27}$ The release of $p_{t-1}^{*}$ is captured by $\omega_{t}=p_{t-1}^{*}-p_{t-1}+\left(s_{t-1}-\lambda_{t-1}\right) q_{t-1}$.

${ }^{28}$ This approximation is exact for $s=0$ or, more generally, for

$$
\int_{p^{\text {mid }}(n)-s}^{p^{\text {mid }}(n)}\left(p^{\text {mid }}(n)-p^{*}\right)^{n} f\left(p^{*}\right) d p^{*}+\int_{p^{\text {mid }}(n)}^{p^{\text {mid }}(n)+s}\left(p^{*}-p^{m i d}(n)\right)^{n} f\left(p^{*}\right) d p^{*}=0 .
$$

${ }^{29}$ See Appendix D for details. 
a right-skewed distribution $f(\cdot)$ with infinite support, namely the halfnormal distribution. Indeed, $p^{\text {mid }}(n)$ increases in $n$, starting from the median for $n=1$, monotonically without bound. If, in contrast, $f(\cdot)$ has finite support, then $p^{\text {mid }}(n)$ increases from the median monotonically toward a finite asymptote $p^{\text {mid }}(\infty) .{ }^{30}$ This is shown in the lower right panel of Figure 5 for the triangular distribution defined on $[0,1]$. We use these observations in the proof of the following proposition:

\section{Proposition 6 (Cross-correlation under market maker information)}

If $\Omega_{t}=\left\{\operatorname{sgn}\left(\varepsilon_{t}\right), p_{t-1}^{*}\right\}$ and Assumption 2 holds, then the optimal market maker response $R\left(\left\{\operatorname{sgn}\left(\varepsilon_{t}\right)\right\}\right)$ strictly increases in risk aversion, $n \geq 1$, without bound. If, further, the distribution of the expected latent price with support $\left[\underline{p}^{*}, \bar{p}^{*}\right]$ satisfies

$$
\left[\frac{\underline{p}_{t}^{*}+\bar{p}_{t}^{*}}{2}-p_{t-1}^{*}\right] \operatorname{sgn}\left(\varepsilon_{t}\right)>s+\frac{\sigma}{E\left(\left|\varepsilon_{t}\right|\right)},
$$

then $\exists n_{0}>1$ such that $\forall n>n_{0}$ it holds that $\operatorname{Corr}\left(\Delta p_{t}^{*}, \Delta u_{t}\right)>0$.

Proof: See Appendix B.6.

Condition (27) holds, for example, for normally distributed $\Delta p_{t}^{*}$, but not for tent distributed $\Delta p_{t}^{*}$.

Comparing these results in the third row of Table 2 with the other model setups, it appears that even though the contemporaneous cross-correlation can be positive for high risk aversion levels, the usual case is that it is negative. For the halfnormal distribution in the upper left panel of Figure 5, for example, we need a rather high risk aversion of $n \geq 8$. Nevertheless, changes in risk aversion of the market maker have a distinctive impact on the cross-correlation. Hansen and Lunde (2006) note as their "Fact IV" that "the properties of the noise have changed over time." Because they base this observation on a comparison of year 2000 with year 2004 it is possible that the underlying cause is a change in risk aversion.

The link between properties of noise and risk aversion offers itself as a way to estimate the time path of risk aversion from the cross-correlation pattern of market prices. In stable periods with low risk aversion the contemporaneous cross-correlation is negative, but as uncertainty shoots up, contemporaneous cross-correlation shoots up with it. In periods of crisis this can lead to the extreme case of an inverted cross-correlation pattern that we have described in this section. The lower row of Figure 4 illustrates this inversion: it shows the typical cross-correlation pattern of strong form efficient prices in a one-period model with modest risk aversion on the left, and under higher risk aversion on the right.

\footnotetext{
${ }^{30}$ Analogously, for left-skewed distributions with infinite support, $p^{\text {mid }}(n)$ decreases in $n$ from the median monotonically without bound, and with finite support toward a finite asymptote $p^{\text {mid }}(\infty)$.
} 
The negative contemporaneous cross-correlation in Hansen and Lunde (2006) indicates that during their sample period the risk aversion of market makers was rather low. ${ }^{31}$

\subsection{Frequent Price Changes}

Until now we discussed models, where the old strong form efficient price becomes public information before any new shift in the latent price. In general, however, the latent price may change again before the old latent price becomes fully publicly known. In this case the old $p_{t-1}^{*}$ still contains unrevealed information about the new $p_{t}^{*}$. As $p_{t-1}^{*}$ is not precisely known itself, potentially the entire history of market prices contains information about $p_{t}^{*}$.

More specifically, suppose that at any point in time the $T$ most recent changes in the strong form efficient price are private information. The signal $\left\{\operatorname{sgn}\left(p_{t}-p_{t}^{*}\right)\right\}$ is now different from the signal $\left\{\operatorname{sgn}\left(\varepsilon_{t}\right)\right\}$. Under the former signal and with $\operatorname{Corr}\left(p_{t}^{*}, \Delta p_{t-\tau}^{*}\right)>0 \forall \tau>0$ the information set $\Omega_{t}$ contains information about $p_{t-\tau}^{*}$ not contained in $\Omega_{t-1}$. By 10 the signs of the cross-correlations at nonzero displacements remain unchanged even if $p_{t}^{*}$ changes frequently. All that is needed is that in expectation market price changes in $t$ are affected by latent price changes in $t-\tau, \tau>0$, by learning. The more often $p_{t}^{*}$ changes during $[t, t-\tau]$, the closer to zero is the cross-correlation $\operatorname{Corr}\left(p_{t}^{*}, \Delta p_{t-\tau}^{*}\right)$, the less informative is the signal in $t$ about $\Delta p_{t-\tau}^{*}$, and thus the closer to zero is the cross-correlation between latent returns and noise. For both signals the contemporaneous cross-correlation is dampened toward zero, because the signal $\left\{\operatorname{sgn}\left(p_{t}-p_{t}^{*}\right)\right\}$ mixes up information on $\Delta p_{t}^{*}$ with information on $\Delta p_{t-\tau}^{*}$, and the signal $\left\{\operatorname{sgn}\left(\varepsilon_{t}\right)\right\}$ is related only to a small component of $\Delta u_{t}$. Overall, slowly decaying private information keeps the cross-correlation sign pattern unchanged, but dampens its absolute values toward zero.

In summary we have shown in this section that many market properties leave their mark on the cross-correlation pattern: The displacement beyond which correlation is zero gives an indication of the frequency of information events. The larger the correlation is in absolute value terms the fewer uninformed trades occur in the market. If contemporaneous strong form cross-correlation is high and positive, then market makers are very risk averse and have access to extra information. If the cross-correlations at nonzero displacements decay

\footnotetext{
${ }^{31}$ Note that in this section from the point of view of the market maker all periods are ex ante identical. Every period the market maker gets the same type of new information $\left(p_{t-1}^{*}\right.$, and either $\operatorname{sgn}\left(\varepsilon_{t}\right)=1$, or $\left.\operatorname{sgn}\left(\varepsilon_{t}\right)=-1\right)$, thus $s_{t}$ is the same in every period. Only a small change in the model allows for time variation in spreads (Demsetz, 1968). If - contrary to the maintained assumptions - we assigned nonzero probability mass to the information event $\left\{\operatorname{sgn}\left(\varepsilon_{t}\right)=0\right\}$ and kept $\operatorname{Var}\left(\varepsilon_{t}\right)=1$ by moving probability mass to the tails of the distribution, then observing this signal would ensure the market maker of no informed trading in this period. Therefore, the competitive spread in this period would be zero. A subsequent $\operatorname{sgn}\left(\varepsilon_{t}\right)= \pm 1$ then would not only trigger a shift in $p_{t}^{\text {mid }}$, but also an increase in spread.
} 
quickly, then market makers learn fast. If they do not decay at all, then informed traders act strategically.

\section{Return/Noise Cross-Correlations in Economic Envi- ronments II: Semi-Strong Efficient Prices}

Now we base the cross-correlation calculation on another latent price, the semi-strong form efficient price, $\tilde{p}_{t}$. Equivalently we could interpret this setup as an endogenous latent price process, determined by an exogenous trading process $q_{t}$, because the strong-form efficient price remains unobserved and enters the model only via the informed trades. This setup is closely related to the generalized Roll (1984) bid-ask model in Hasbrouck (2007).

\subsection{The General Multi-Period Case}

In the period of a change in the strong form efficient price, in which also the previous strong form efficient price becomes public information, the semi-strong form efficient return is ${ }^{32}$

$$
\Delta \tilde{p}_{0}^{e}=\lambda_{0} q_{0}+\sigma \varepsilon_{-T}-\sum_{i=1}^{T}\left(\lambda_{-i} q_{-i}+\omega_{-i}\right)+\omega_{0}
$$

where the first term reflects the market maker's guess about the new strong form efficient return based on a trade, the second term internalizes the new information about the previous return, and as a countermove the sum undoes the now obsolete guesses about the previous return. In all other periods the semi-strong form efficient price changes by

$$
\Delta \tilde{p}_{t}^{e}=\lambda_{t} q_{t}+\omega_{t}
$$

From (8) we get for $\forall t$

$$
\Delta u_{t}=\left(s_{t}-\lambda_{t}\right) q_{t}-\left(s_{t-1}-\lambda_{t-1}\right) q_{t-1}
$$

where the first two terms reflect information-revealing trades, and the second two terms reflect the bid-ask bounce.

Using Assumption 1 this immediately leads to an expression for the contemporaneous covariance $^{33}$

\footnotetext{
${ }^{32}$ We use again the shorthand notation $p_{0} \equiv p_{\kappa T} \forall \kappa \in \mathbb{Z}$, and likewise $p_{-x} \equiv p_{\kappa T-x} \forall \kappa, x \in \mathbb{Z}$.

${ }^{33}$ To keep the terms manageable, we assume no extra information here, i.e. $\omega_{t}=0 \forall t$.
} 


$$
\begin{aligned}
\operatorname{Cov}\left(\Delta \tilde{p}_{t}^{e}, \Delta u_{t}\right) & =\frac{1}{T}\left\{-\phi_{0} \lambda_{0}\left(\lambda_{0}-s_{0}\right)+\sigma\left(\lambda_{-1}-s_{-1}\right) E\left(q_{-1} \varepsilon_{-T}\right)\right. \\
& -\sum_{i=-1}^{-T}\left(\lambda_{-1}-s_{-1}\right) \lambda_{i} E\left(q_{i} q_{-1}\right) \\
& \left.+\sum_{i=1}^{T-1}\left(-\phi_{i} \lambda_{i}\left(\lambda_{i}-s_{i}\right)+\lambda_{i}\left(\lambda_{i-1}-s_{i-1}\right) E\left(q_{i} q_{i-1}\right)\right)\right\},
\end{aligned}
$$

for covariance at higher displacements $\tau \in[1, T-1]$

$$
\begin{aligned}
& \operatorname{Cov}\left(\Delta \tilde{p}_{t-\tau}^{e}, \Delta u_{t}\right)=\frac{1}{T}\left\{-\lambda_{0}\left(\lambda_{\tau}-s_{\tau}\right) E\left(q_{0} q_{\tau}\right)\right. \\
& +\lambda_{0}\left(\lambda_{\tau-1}-s_{\tau-1}\right) E\left(q_{0} q_{\tau-1}\right)+\lambda_{T-\tau}\left(\lambda_{T-1}-s_{T-1}\right) E\left(q_{T-\tau} q_{T-1}\right) \\
& \left.+\sum_{i=\tau+1}^{T-1}\left[\lambda_{i-\tau}\left(-\lambda_{i}+s_{i}\right) E\left(q_{i-\tau} q_{i}\right)+\lambda_{i-\tau}\left(\lambda_{i-1}-s_{i-1}\right) E\left(q_{i-\tau} q_{i-1}\right)\right]\right\}
\end{aligned}
$$

for covariance at displacement $T$

$$
\operatorname{Cov}\left(\Delta \tilde{p}_{t-T}^{e}, \Delta u_{t}\right)=\frac{1}{T} \lambda_{0}\left(\lambda_{T-1}-s_{T-1}\right) E\left(q_{0} q_{T-1}\right)
$$

and for all higher order displacements $\tau>T$

$$
\operatorname{Cov}\left(\Delta \tilde{p}_{t-\tau}^{e}, \Delta u_{t}\right)=0
$$

Under semi-strong market efficiency $\left(s_{t}=\lambda_{t} \forall t\right)$ the cross-correlation function is zero for all displacements. Hence all of the special cases that we now discuss assume lack of even this weak form of market efficiency.

\subsection{Special Multi-Period Cases}

The cross-correlations for semi-strong form efficient prices stem from a gap between the spread, $s_{t}$, and the adverse selection parameter, $\lambda_{t}$. Such a gap can result from processing costs $\left(s_{t}>\lambda_{t}\right)$, from legal restrictions $\left(s_{t}<\lambda_{t}\right)$, or merely from suboptimal behavior of the market maker. Noisy signals or strategic behavior do not affect the semi-strong crosscorrelations - all what matters is that the market maker's knowledge passes into market prices one-to-one.

In Easley and O'Hara (1992), for example, prices are semi-strong form efficient by definition, and therefore the semi-strong form cross-correlation function is zero always. 
The Kyle (1985) model assumptions $\lambda_{t}=\lambda$ and $s_{t}=s \forall t$ give with (30)

$$
\operatorname{Cov}\left(\Delta \tilde{p}_{t-\tau}^{e}, \Delta u_{t}\right)=\frac{\lambda(\lambda-s)}{T}\left\{E\left(q_{T-\tau} q_{T-1}\right)+\sum_{i=\tau}^{T-1}\left[E\left(q_{i-\tau} q_{i-1}\right)-E\left(q_{i-\tau} q_{i}\right)\right]\right\} .
$$

If $\lambda=0$, then this cross-correlation is flat at zero. Likewise, if additionally $E\left(q_{i-\tau} q_{i}\right)$ is a positive constant between the time of the latent price change and its announcement, the cross-correlation is flat and proportional to $\frac{\lambda(\lambda-s)}{T}$. More generally, because typically $E\left(q_{i} q_{j}\right)>E\left(q_{i-\tau} q_{j}\right)>0 \forall i \leq j, \forall \tau>0$, the cross-correlation decreases in $\tau$.

\subsection{One-Period Case}

The simpler case of markets in which all information is revealed after one period without any extra information, i.e.

$$
\Delta \tilde{p}_{t}^{e}=\lambda\left(q_{t}-q_{t-1}\right)+\sigma \varepsilon_{t-1}
$$

offers itself again for illustration of these cross-correlation effects. In the one period case the semi-strong form efficient prices follow a martingale, but unlike their strong form counterpart the semi-strong form efficient returns do not follow a martingale difference sequence. ${ }^{34}$ We see in the following proposition that in contrast to the strong form correlations, the absolute value of semi-strong form cross-correlation at displacement zero and one usually differs.

\section{Proposition 7 (Semi-strong form cross correlation, one-period model)}

The contemporaneous cross-correlation is

$$
\operatorname{Corr}\left(\Delta \tilde{p}_{t}^{e}, \Delta u_{t}\right)=\frac{2 \phi \lambda-\sigma E\left(q_{t} \varepsilon_{t}\right)}{\sqrt{\sigma^{2}-2 \sigma \lambda E\left(q_{t} \varepsilon_{t}\right)+2 \phi \lambda^{2}}} \frac{\operatorname{sgn}(s-\lambda)}{\sqrt{2 \phi}}
$$

The cross-correlation at displacement one equals

$$
\operatorname{Corr}\left(\Delta \tilde{p}_{t-1}^{e}, \Delta u_{t}\right)=\frac{-\phi \lambda}{\sqrt{\sigma^{2}-2 \sigma \lambda E\left(q_{t} \varepsilon_{t}\right)+2 \phi \lambda^{2}}} \frac{\operatorname{sgn}(s-\lambda)}{\sqrt{2 \phi}}
$$

All cross-correlations at higher displacements are zero.

Proof: See Appendix B.7.

Bounds on the contemporaneous cross-correlation can be obtained by assuming a specific market marker loss function and then solving for the market maker's optimal $\lambda$. For example,

\footnotetext{
${ }^{34}$ In multiperiod models strong form efficient prices follow a martingale, but semi-strong form efficient prices do not.
} 
suppose the market maker has a quadratic loss function, then

$$
\lambda^{o p t}=\underset{\lambda}{\operatorname{argmin}} E\left[\left(\tilde{p}_{t}^{e}-p_{t}^{*}\right)^{2}\right],
$$

which becomes

$$
\lambda^{o p t}=\underset{\lambda}{\operatorname{argmin}} \phi \lambda^{2}-2 \sigma \lambda E\left(q_{t} \varepsilon_{t}\right),
$$

and therefore $\lambda^{o p t}=\frac{\sigma}{\phi} E\left(q_{t} \varepsilon_{t}\right)>0$. At $\lambda^{o p t}$ we have

$$
\begin{gathered}
\operatorname{Corr}\left(\Delta \tilde{p}_{t}^{e}, \Delta u_{t}\right)=E\left(q_{t} \varepsilon_{t}\right) \frac{\operatorname{sgn}\left(s-\lambda^{o p t}\right)}{\sqrt{2 \phi}}, \\
\operatorname{Corr}\left(\Delta \tilde{p}_{t-1}^{e}, \Delta u_{t}\right)=-E\left(q_{t} \varepsilon_{t}\right) \frac{\operatorname{sgn}\left(s-\lambda^{o p t}\right)}{\sqrt{2 \phi}},
\end{gathered}
$$

and because $0 \leq E\left(q_{t} \varepsilon_{t-\tau}\right)<1, \forall t, \forall \tau .^{35}$

$$
\left|\operatorname{Corr}\left(\Delta \tilde{p}_{t}^{e}, \Delta u_{t}\right)\right|=\left|\operatorname{Corr}\left(\Delta \tilde{p}_{t-1}^{e}, \Delta u_{t}\right)\right| \leq \frac{1}{\sqrt{2 \phi}}
$$

Under a quadratic market maker loss function and an uninterrupted flow of trades $(\phi=1)$, the absolute value of cross-correlations is bounded from above by $\frac{1}{\sqrt{2}}$.

Proposition 7 shows that the size of the spread matters only relative to the adverse selection parameter. The cross-correlation at displacement one, for example, is negative if and only if the spread exceeds the adverse selection cost. $s>\lambda$ is reasonable, because the spread must cover the order processing cost. It also entails, however, that the average trader in expectation incurs a loss with every transaction. Hasbrouck (2007) justifies this with the liquidity needs of traders.

The sign of contemporaneous cross-correlation is ambiguous in general. As in Diebold (2006), no matter what loss function applies, for $s$ sufficiently large and $\lambda>\frac{\sigma}{2 \phi} E\left(q_{t} \varepsilon_{t}\right)=\frac{\lambda_{o p t}}{2}$ the model predicts a cross-correlation pattern that is the opposite of the empirical pattern in Hansen and Lunde (2006). We illustrate this again in the last row of Figure 4, which on the left shows the cross-correlation function for a relatively small spread $(0 \leq s<\lambda)$, and on the right for a typical, wider spread $(s>\lambda>0)$. If sufficiently many lags are included, the Hansen and Lunde estimator is unbiased for the strong form efficient price defined as in (1) and (2), but by construction not for its semi-strong form counterpart.

\footnotetext{
${ }^{35}$ See equation $\sqrt{38}$ in Appendix A.2.
} 
All it takes to generate such a large spread without violating the market maker's zeroprofit condition is high risk aversion. By the same reasoning as in section $3.3 .2,{ }^{36}$ there exists a minimal risk aversion level $n_{0}$ such that all $n>n_{0}$ generate a spread $s>\lambda$. Thus if additionally $\lambda>\frac{\sigma}{2 \phi} E\left(q_{t} \varepsilon_{t}\right)$, then there exists $n_{0}$ such that all $n>n_{0}$ generate a positive contemporaneous cross-correlation and a negative cross-correlation at displacement one. Note that unlike for the strong-form efficient prices discussed in section 3.3 .2 positive contemporaneous cross-correlation for semi-strong form efficient prices obtains even though the market maker does not observe a signal. We summarize the results in the lower four rows of Table 2 ,

Summing up, what sign of contemporaneous cross-correlation does market microstructure theory predict? Positive contemporaneous cross-correlations occur for (1) strong form efficient prices under sufficiently high risk aversion if a signal is observed, and (2) semi-strong form efficient prices for large spreads. Various market arrangements and sampling speeds can dampen the contemporaneous cross-correlation to zero, but the negative sign maintains except in the two aforementioned cases. Bandi and Russell (2006) and Diebold (2006) rightly wonder whether a negative cross-correlation is inevitable. In contrast to Hansen and Lunde (2006), Bandi and Russell (2008) find no "obvious evidence of a significant, negative correlation." These seemingly contradictory results might stem from the inability of purely statistical estimators to clearly distinguish strong form from semi-strong form efficient prices. Without controlling for market features, which the realized volatility literature so far largely ignores, the estimate may pick up any of the two prices. As we have seen, a positive crosscorrelation is possible, but a negative cross-correlation appears most realistic for strong form efficient prices.

\section{Additional Discussion}

We have already drawn some econometric implications insofar as we have shown that market microstructure models predict rich cross-correlation patterns between latent prices and microstructure noise, which have yet to be investigated empirically. Here we go farther, sketching some specific aspects of such empirics, including the relationship between theory-based and data-based (sample) cross-correlation functions, strategies for using microstructural information to obtain improved "structural" volatility estimators, and comparative aspects of structural and non-structural volatility estimators.

\footnotetext{
${ }^{36}$ The spread affects the market maker price conditional on the trade direction, thus the market maker belief conditional on the trade direction is truncated on one side at $p_{t}^{e}$.
} 


\subsection{Effects of Sampling Frequency on Return/Noise Correlations}

We have thus far focused on sampling at the rate corresponding exactly to the market maker's reaction time. Sampling at faster or slower rates will affect the shape of cross-correlation functions. This has immediate implications for the shape of empirically estimated (sample) cross-correlation functions, because the reaction speed of the market maker is generally unknown, so that econometric sampling may proceed at faster or slower rates.

Consider first the effects of sampling "too fast." Suppose we sample $m$ times during an interval of no changes in both latent and market prices, and record each time the most recently realized prices. Then all returns except for the first one are zero and thus the cross-correlation function becomes a spread-out version of the cross-correlation functions derived in the previous sections: after each non-zero cross-correlation follow $m-1$ zero cross-correlations, as in the second row of Table 3 . Zeros in the middle of a cross-correlation function thus indicate overly fast sampling.

Table 3: Cross-Correlation Patterns at Various Sampling Frequencies

\begin{tabular}{|c|c|c|c|c|c|c|c|}
\hline \multirow[t]{2}{*}{ sampling rate } & \multicolumn{6}{|c|}{ cross-correlation function } & \multirow{2}{*}{ note } \\
\hline & $\tau=0$ & 1 & 2 & 3 & 4 & 5 & \\
\hline optimal & $\rho_{0}$ & $\rho_{1}$ & $\rho_{2}$ & $\rho_{3}$ & $\rho_{4}$ & $\rho_{5}$ & \\
\hline$>$ latent price frequency & $\rho_{0}$ & 0 & 0 & $\rho_{1}$ & 0 & 0 & \\
\hline$>$ market maker update freq. & $\rho_{0}^{M M}$ & 0 & $\rho_{1}^{M M}$ & 0 & $\rho_{2}^{M M}$ & 0 & $\rho_{i}^{M M}>\rho_{i} \forall i$ \\
\hline$<$ latent price frequency & $\rho_{0}^{S L}$ & $\rho_{1}^{S L}$ & $\rho_{2}^{S L}$ & $\rho_{3}^{S L}$ & $\rho_{4}^{S L}$ & $\rho_{5}^{S L}$ & $\rho_{i}^{S L}<\rho_{i} \forall i$ \\
\hline
\end{tabular}

A variant of sampling "too fast" is sampling exactly at trading frequency, but with a market maker in the background who updates $p_{t}^{e}$ infrequently, for example only every second period. After a latent price change at $t=0$, he updates his quotes for the first time at $t=2$, and then, observing the trades in between, again at $t=4, t=6$, and so forth. The expected noise pattern is therefore $-\Delta p_{t}^{*}, 0, \Delta p_{2}, 0, \Delta p_{4}, \ldots$ Trading activity in the interim period provides information about $\Delta p_{t}^{*}$ and thus $\Delta p_{2}$ is more correlated with $\Delta p_{t}^{*}$ than under period-by-period updating. But because the quote in the interim period is fixed, the two periods together provide less additional information than under period-by-period updating. Whereas the variance of $\Delta p_{t}^{*}$ is unchanged, the (unconditional) variance of noise shrinks to somewhat more than half the variance that obtains when the market maker updates $p_{t}$ every period. The cross-correlation function therefore oscillates, as in the third row of Table 3 .

Now consider the effects of sampling "too slowly". Suppose, for example, that in the one-period model of section 4.3 we sample only every $n$-th tick, where $\hat{t}$ indexes the $n$-tick 
blocks. Then (32) becomes

$$
\Delta \tilde{p}_{\hat{t}}^{e}=\sum_{i=(\hat{t}-1) n+1}^{\hat{t}_{n}} \Delta \tilde{p}_{i}^{e}=\lambda\left(q_{\hat{t} n}-q_{(\hat{t}-1) n}\right)+\sigma \sum_{i=(\hat{t}-1) n}^{\hat{t}^{n}-1} \varepsilon_{i},
$$

and the variance increases to $\operatorname{Var}\left(\Delta p_{\hat{t}}^{e}\right)=2 \phi \lambda^{2}+n \sigma^{2}-2 \lambda \sigma E\left(q_{t} \varepsilon_{t}\right)$. Assuming that the statistical properties of the interim periods are the same as the properties of the sampled periods, the expressions for noise (28), its variance $\operatorname{Var}\left(\Delta u_{\hat{t}}\right)$, and the covariance $\operatorname{Cov}\left(\Delta \tilde{p}_{\hat{t}}^{e}, \Delta u_{\hat{t}}\right)$ remain unchanged. Thus

$$
\left|\operatorname{Corr}\left(\Delta \tilde{p}_{\hat{t}}, \Delta u_{\hat{t}}\right)\right|=\left|\frac{2 \phi \lambda-\sigma E\left(q_{t} \varepsilon_{t}\right)}{\sqrt{2 \phi} \sqrt{n \sigma^{2}-2 \lambda \sigma E\left(q_{t} \varepsilon_{t}\right)+2 \phi \lambda^{2}}}\right|<\left|\operatorname{Corr}\left(\Delta \tilde{p}_{t}, \Delta u_{t}\right)\right|,
$$

so that increasing the sampling interval averages the initial market price reaction with later price changes, thereby dampening the entire cross-correlation pattern toward zero. The most informative cross-correlations are therefore obtained by sampling sufficiently fast to capture every tick separately. Hansen and Lunde (2006) find a negative contemporaneous crosscorrelation between returns and noise, which diminishes as more ticks are combined into one market price sample. Our results show that this can stem from two different sources: Either from the averaging effect across latent price changes just described, or from crosscorrelations at nonzero displacements offsetting the contemporaneous correlation for the same latent price change. This ambiguity can be resolved by evaluating the entire crosscorrelation function, especially its cutoff, which shows the importance of not limiting noise analysis to the contemporaneous cross-correlation.

The upshot is that sampling frequency does not change the sign pattern of cross-correlations but can severely impact their absolute values, as summarized in Table 3 . At low sampling rates the cross-correlations become empirically indistinguishable from zero, whereas at higher sampling frequencies the cross-correlation structure of the noise needs to be addressed. In the next section we suggest strategies for doing so in parsimonious fashion by exploiting market microstructure theory.

\subsection{Structural Volatility Estimation via Microstructural Restric- tions}

In the introduction we highlighted the key issue of estimation of integrated volatility $(I V)$ using high-frequency data, the potential problems of the first-generation estimator (simple realized volatility $-R V$ ) in the presence of MSN, and subsequent attempts to "correct" for 
MSN.

In an important development, Hansen and Lunde (2006) suggest making $R V$ robust to serial correlation via HAC estimation methods, which are asymptotically justified under very general conditions. That asymptotic generality is, however, not necessarily helpful in finite samples. Indeed the frequently unsatisfactory finite-sample performance of nonparametric HAC estimators leads Bandi and Russell (2010) to suggest sophisticated alternative statistical approaches.

Here we explore aspects of a different approach that specializes the estimator in accordance with the implications of market microstructure theory. As we have seen, dynamic market microstructure models imply that noise decays geometrically over time after displacement one, with two polar cases of immediate decay (as in section 3.3) and no decay (as in section 3.2.2. That knowledge can be used to construct volatility estimators that exploit the restrictions implied by market microstructure theory ${ }^{37}$

Consider first a "bid-ask bounce estimator", based on a one-period model without extra information and constant spread. The only action in the market price is then the bid-ask bounce. Suppose we are interested in the volatility of the strong form efficient price. From (4) and (5) we obtain $\Delta p_{t}=\Delta p_{t}^{*}+\Delta u_{t}$ with $\Delta u_{t}=\sigma\left(\varepsilon_{t-1}-\varepsilon_{t}\right)+s\left(q_{t}-q_{t-1}\right)$. It follows that

$$
E\left[\left(\Delta p_{t}^{*}\right)^{2}\right]=E\left[\left(\Delta p_{t}-\Delta u_{t}\right)^{2}\right]=E\left(\Delta p_{t}^{2}\right)+2 s\left(\sigma E\left(q_{t} \varepsilon_{t}\right)-\phi s\right) .
$$

Simple calculations reveal that the last term equals twice the first-order autocorrelation of market returns, so that, even if $E\left(q_{t} \varepsilon_{t}\right) \neq 0$, a consistent estimator for $I V$ of the strong form efficient price is ${ }^{38}$

$$
\widehat{I V}=R V+2 E\left(\Delta p_{t} \Delta p_{t-1}\right)
$$

It is interesting to note the resemblance to the Roll (1984) estimator.

As another example, consider a "learning estimator". Suppose $\Delta p_{t}$ follows an $\mathrm{MA}(\infty)$ process in the innovations for the latent price,

$$
\Delta p_{t}=\sigma\left(\alpha_{1} \varepsilon_{t}+\sum_{\tau=1}^{\infty} \alpha_{2}^{\tau} \varepsilon_{t-\tau}\right) .
$$

\footnotetext{
${ }^{37}$ We do not claim optimality; instead we show the practical relevance of tailoring the estimator to the market at hand. As emphasized before, optimality of a $R V$ estimate must be defined relative to the user's objective.

${ }^{38}$ Hasbrouck (1993) and recently Hansen et al. (2008) show how to embed (33) into general moving average (MA)-based estimators. Such general MA-estimators are warranted if the researcher has only limited information about the microstructure of the market or has interest different from $I V$ estimation, such as forecasting the latent price process. If, however, the microstructure is known and interest centers on estimating $I V$, as we assume here, then our estimator may be more appealing.
} 
This form of $\Delta p_{t}$ accommodates very persistent cross-correlations, similar to the idea behind the sequence of examples in Oomen (2006). If our knowledge of the market is this comprehensive, we can obtain a consistent estimate for $I V=\sigma^{2}$ from (34) in a GMM framework using three moments. ${ }^{39}$ For example, a learning model with $T=\infty$ and frequent latent price changes might predict

$$
\Delta p_{t} \approx 0 \cdot \sigma \varepsilon_{t}+\sum_{\tau=1}^{\infty}\left(-e^{-r \tau}+e^{-r(\tau-1)}\right) \sigma \varepsilon_{t-\tau}=\sigma\left(e^{r}-1\right) \sum_{\tau=1}^{\infty} e^{-r \tau} \varepsilon_{t-\tau}
$$

The $I V\left(=\sigma^{2}\right)$ can then be consistently estimated directly by GMM. The resulting estimate of $I V$ is a scaled version of standard $R V^{40}$

$$
\widehat{I V}=\frac{1-e^{-2 \hat{r}}}{1-2 e^{-\hat{r}}+e^{-2 \hat{r}}} \cdot R V
$$

where the scaling factor requires a consistent estimator of only one parameter, the market maker's learning rate, $r$.

The expression for $\widehat{I V}$ in $(35)$ offers a structural interpretation to estimates of noise and $I V$, such as those in Table 3 of Hansen and Lunde (2006). The model of Easley and O'Hara (1992) predicts that the noise decreases with the learning rate. Slow learning implies a very persistent cross-correlation between noise and latent returns, and hence persistent autocorrelation of noise, so that fluctuations in noise tend to dominate the $I V .^{41}$

Figure 6 provides some perspective. It is based on the noise-to- $I V$ ratios reported by Hansen and Lunde (2006), which are (unfortunately) derived under the assumption of independent noise. The ratio of noise to $I V$ shrinks with the number of price-changing quotes per day. If the number of times that the market maker changes his price quote during a trading day is indicative of his speed of learning, then MSN indeed decreases as the learning rate of the market maker increases. Thus, even though these ratios may not be directly applicable, they seem to support the multiperiod learning model.

Furthermore, the recent decline in noise-induced bias of $R V$ (Hansen and Lunde's fact III) suggests that the learning rate $r$ has increased. Meddahi's (2002) finding that the standard

\footnotetext{
${ }^{39}$ The proof, which we sketch here, is straightforward. Recast the price process $(1)$ and $(2)$ in continuous time, so that $\Delta p_{t}=\Delta p_{t}^{*}+\Delta u_{t} / \sqrt{m}$, with $m$ denoting the number of subintervals, $t m$ equal to one unit of calendar time, and the scale of $t$ suitably redefined. Then under standard assumptions $r$ is invariant to $m$ and local infill asymptotic theory can be applied.

${ }^{40}$ Hansen et al. (2008) present an estimator of $I V$ which, like (35), is a scaled variant of $R V$. The key difference between the two estimators is that we exploit (that is, condition on) the relevant market microstructure, whereas Hansen et al. (2008) attempt to achieve robustness to a wide range of possible microstructures.

${ }^{41}$ Kelly and Steigerwald (2004) take this a step further by linking information persistence and frequency with the difference between prices and trades in the decay rate of autocorrelations.
} 
deviation of the bias is large relative to the $I V$ suggests that the learning rate itself may have fluctuated considerably around its increasing trend.

Figure 6: Ratio of Noise to Integrated Variance, as a Function of Quotes per Day

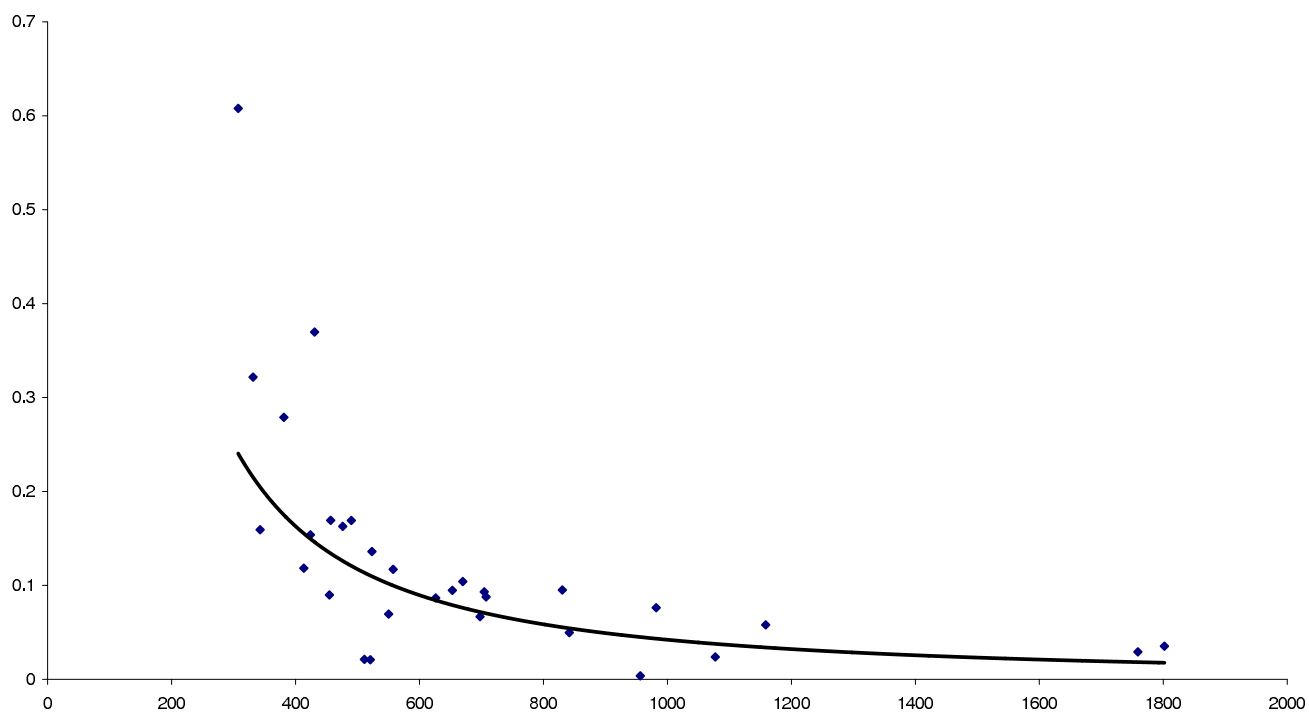

Notes: The vertical axis measures the noise-to-signal ratio as 100 times noise divided by $I V$ under the assumption of independent noise. The horizontal axis gives the number of quotes per day with a price change. Data are for 30 NYSE and NASDAQ equities in 2000, obtained from Hansen and Lunde (2006) Tables 1 and 3. The solid line is a fitted trend.

Table 4 reveals that both the learning and bid-ask estimators explain at least one third of noise. Learning appears to be very fast for the Alcoa stock (high $r$ ), which implies quick decay of the MA terms, such that essentially only the MA(1) term matters. As a result the learning and bid-ask volatility estimates are very similar. Based on this evidence, the MA(1) captures both the learning and bid-ask bounce effects. ${ }^{42}$ These are the two most common microstructure effects, which we indeed want to remove. But what about the other two thirds of the "noise"? It is not clear whether the difference between the bidask and Hansen-Lunde estimates, say, is really "noise", unless one has in mind a distorting microstructure effect other than learning and bid-ask bounce. Hence our perspective warns against removing "noise" from a volatility estimate when the noise does not have a plausible noise interpretation.

\footnotetext{
${ }^{42}$ All estimators in the table except the standard estimator allow for correlation between noise and latent price. The bid-ask bounce we remove thus includes both uninformed and informed components. The uninformed component alone can be calculated by taking $s$ directly from the difference between bid and ask in the data, and $\phi$ as the number of seconds with a trade or quote.
} 
Table 4: Comparison of Realized Volatility Estimators

\begin{tabular}{c|cccc}
\hline$R V$ estimator & price & mid & bid & ask \\
\hline$R V_{\text {Standard }}$ & 2.487 & 1.601 & 2.726 & 2.679 \\
$R V_{\text {Learning }}$ & 2.373 & 1.544 & 2.525 & 2.477 \\
$R V_{\text {Bid-ask }}$ & 2.370 & 1.543 & 2.517 & 2.468 \\
$R V_{\text {Hansen-Lunde }}$ & 2.142 & 2.138 & 2.250 & 2.253 \\
$R V_{\text {Semi-strong }}$ & 2.258 & 1.517 & 2.352 & 2.297 \\
\hline
\end{tabular}

Notes: The price data are second-by-second for Alcoa Inc. (AA) in 2004, from Barndorff-Nielsen et al. (2009). $R V_{\text {Standard }}$ is simple realized volatility, $\Delta p_{t}^{2} . R V_{\text {Learning }}$ is the strong form efficient price estimator under asymmetric information, and $R V_{B i d-a s k}$ is the strong form efficient price estimator accounting for a non-zero spread, as described in section 5.2, $R V_{\text {Hansen-Lunde }}$ is the statistical estimator of Hansen and Lunde (2006). $R V_{\text {Semi-strong }}$ is the semi-strong form efficient price estimator as defined in section 5.3 .

\subsection{On Structural vs. Non-Structural Volatility Estimators}

Here we emphasize that the more the econometrician knows about the price process of relevance, the more the noise correction can be tailored to it by exploiting microstructure theory. This is important, because as discussed in section 2 , the price process of interest may differ across users of volatility estimates (e.g., many users are likely to be interested in price processes different from (1), which has implications for appropriate volatility estimation. The estimate of the volatility of strong form efficient return, 20), the theoretical price under full information, differs both conceptually and numerically from the volatility of semi-strong form efficient returns,

$$
E\left[\left(\Delta \tilde{p}_{t}^{e}\right)^{2}\right]=\frac{1}{T}\left\{\sigma^{2}+\sum_{i=0}^{T-1} \phi_{i} \lambda_{i}^{2}+E\left[\left(\sum_{i=-1}^{-T} \lambda_{i} q_{i}\right)^{2}\right]-2 \sigma \sum_{i=-1}^{-T} \lambda_{i} E\left(q_{i} \varepsilon_{-T}\right)\right\}
$$

which is the volatility that affects the balance sheet of the market maker. Thus studies of market maker behavior should be based on a RV estimate $E\left[\left(\Delta \tilde{p}_{t}^{e}\right)^{2}\right]$, not $E\left[\left(\Delta p_{t}^{*}\right)^{2}\right]$. For example, consider again $T=1$, in which case strong form volatility 20 is $\sigma^{2}$ and semi-strong volatility 36 simplifies to

$$
E\left[\left(\Delta \tilde{p}_{t}^{e}\right)^{2}\right]=\sigma^{2}+2 \phi \lambda^{2}-2 \sigma E\left(q_{t} \varepsilon_{t}\right) \neq \sigma^{2}
$$

But the RV estimator promoted by Hansen and Lunde (2006) is

$$
R V_{A C_{1}}^{1 t i c k}=\Delta p_{t}^{2}+\Delta p_{t-1} \Delta p_{t}+\Delta p_{t} \Delta p_{t+1},
$$


which for $T=1$ is

$$
E\left(R V_{A C_{1}}^{1 t i c k}\right)=E\left[\left(s\left(q_{t}-q_{t-1}\right)+\sigma \varepsilon_{t-1}\right) \times\left(\sigma\left(\varepsilon_{t}+\varepsilon_{t-1}+\varepsilon_{t-2}\right)+s\left(q_{t+1}-q_{t-2}\right)\right)\right]=\sigma^{2} .
$$

Hence although $R V_{A C_{1}}^{1 t i c k}$ is unbiased for $\sigma^{2}$, it is in general biased with ambiguous direction for $\operatorname{Var}\left(\Delta \tilde{p}_{t}^{e}\right)$, because by construction a noise-robust estimator with lag window $T$ correctly removes any microstructure and other correlation effects. For this estimator to work, the latent price process of interest must follow a martingale difference sequence (MDS). Even though semi-strong form prices with $T=1$ form a martingale, their returns are not an MDS. They are serially correlated and inevitably $R V_{A C_{1}}^{1 t i c k}$ is biased relative to $\operatorname{Var}\left(\Delta \tilde{p}_{t}^{e}\right)$.

Consider, for example, a "semi-strong form efficient price estimator". We work in a oneperiod model without extra information and constant spread, but we are now interested in the volatility of the semi-strong form efficient price. From (4) and (5), using (32), we obtain $\Delta p_{t}=\Delta \tilde{p}_{t}^{e}+\Delta u_{t}$ with $\Delta u_{t}=\sigma\left(\varepsilon_{t-2}-\varepsilon_{t-1}\right)+s\left(q_{t}-q_{t-1}\right)+\lambda\left(-q_{t}+2 q_{t-1}-q_{t-2}\right)$. It follows that

$$
E\left[\left(\Delta \tilde{p}_{t}^{e}\right)^{2}\right]=E\left(\Delta p_{t}^{2}\right)+2 s \phi(\lambda-s) .
$$

One can show that the last term equals twice the difference of the first- and second-order autocorrelations of market returns; hence a consistent estimator for $I V$ of the semi-strong form efficient price is

$$
\widehat{I V}=R V+2\left[E\left(\Delta p_{t} \Delta p_{t-1}\right)-E\left(\Delta p_{t} \Delta p_{t-2}\right)\right]
$$

The obvious difference between (33) and (37) emphasizes the importance of carefully defining the latent price series of interest. Note also that whereas sampling only every second price would remove all microstructure noise from the $R V$ estimate of the strong form efficient price in (33) (and thus $\widehat{I V}^{\text {bae }}=R V$ ), it would not remove all noise from the $R V$ estimate of the semi-strong form efficient price (37), because $E\left(\Delta p_{t} \Delta p_{t-2}\right)=s\left(\sigma E\left(q_{t} \varepsilon_{t}\right)-\phi \lambda\right) \neq 0$.

We show estimates for the volatility of semi-strong form efficient prices in the bottom row of Table 4. The strong form efficient price estimator (i.e., the bid-ask estimator) would overstate the volatility of semi-strong form efficient prices by five percent or more. This emphasizes quantitatively the potential importance of tailoring the noise correction to the latent price of interest.

In this paper we model the strong form efficient price as an MDS, and indeed this latent price series is of interest on its own. We doubt, however, that this is the unique latent price of interest in volatility estimation. Efficient prices from an informed trader's perspective 
could themselves be seen as the result of a learning process about the state of the economy, ${ }^{43}$ which implies that the $p_{t}^{*}$ of interest is often not an MDS, but instead has the properties that we have derived in this paper for the semi-strong form efficient price $\tilde{p}_{t}$.

Suppose, for example, that the strong form efficient prices violate (2) and are themselves the result of learning by informed traders about fundamentals, $\eta_{t}$, which follow a random walk,

$$
\Delta p_{t}^{*}=\sigma \sum_{\tau=1}^{T}\left(-e^{-r_{1} \tau}+e^{-r_{1}(\tau-1)}\right) \eta_{t-\tau} .
$$

Suppose as well that market prices follow the usual process of market maker learning,

$$
\Delta p_{t}=\sum_{\tau=1}^{T}\left(-e^{-r_{2} \tau}+e^{-r_{2}(\tau-1)}\right) \Delta p_{t-\tau}^{*}
$$

Then mechanically calculating $R V_{A C(T)}^{1 t i c k}$ gives the variance of the fundamental, not the variance of the strong form efficient price. Obviously, a purely statistical noise correction cannot distinguish between cross-correlation caused by fundamentals and cross-correlation caused by MSN. ${ }^{44}$ This is where market microstructure theory can contribute new insights to $R V$ estimation. By providing distinctive but flexible relationships between noise and latent returns, we can decompose the agnostic statistical noise estimate into its various components, such as MSN and fundamental correlation in the strong form efficient price (in our example discussed previously). Our example uses a MA(2T) process with only two free coefficients, but the large sample sizes typical with high frequency data can accommodate much richer specifications. Empirical work in market microstructure tends to favor extreme parametrizations, ranging from the very parsimonious as in the regressions of Glosten and Harris (1988), to the profligate as in the vector autoregressions of Hasbrouck (1996). For RV noise correction the most useful parametrizations may be intermediate, imposing a general correlation pattern but avoiding highly situation-specific assumptions.

\footnotetext{
${ }^{43} \mathrm{Also}$, they might be the result of learning about information of other market participants, as in Foster and Viswanathan (1996).

${ }^{44}$ Standard $R V$ is unbiased if sampling frequency is sufficiently low so that microstructure effects are averaged out. In a volatility signature plot for our dataset (see the upper panels for 2004 of Figure 1 in Hansen and Lunde (2006), up to 30 minute sampling cycle duration) the standard $R V$ seems to decline with sampling frequency. $R V_{A C N W(30)}$ deviates considerably from the low frequency level of standard $R V$, and thus whereas it might remove high-frequency noise, it appears biased at small frequencies, at least in small samples. Thus "noise-corrected" $R V$ estimators should only be applied to data sampled at frequencies at which microstructure effects can conceivably exist, e.g. above 0.01 per second. Applying them to data at lower frequencies results in biased estimates, because at lower frequencies slow moving features of the price process are removed, not microstructure noise.
} 


\section{Concluding Remarks}

The recent realized volatility literature provides statistical insights into microstructure noise (MSN) and its effects. In this paper we have provided complementary economic insights, treating MSN not simply as a nuisance, but rather as the result of financial economic decisions, which we seek to understand. ${ }^{45}$ In that regard, we derived the predictions of economic theory regarding correlation between MSN and two types of latent price, characterizing and contrasting the entire cross-correlation functions in a variety of market environments, with a variety of results.

Some results are generic. For example, cross-correlations between strong form efficient price and MSN at displacements greater than zero have sign opposite to that of the contemporaneous correlation.

Some results are not generic but nevertheless quite robust to model choice. For example, all models predict negative contemporaneous correlation between latent price and MSN, so long as the risk aversion of market makers is not too high.

Finally, some results are highly model-specific. For example, the cross-correlation patterns and absolute magnitudes depend critically on the frequency of latent price changes, the presence of bid/ask bounce, the timing of information and actions, and the degree of market maker risk aversion.

We hope that the results of this paper will promote the use of theory in disciplining data. We have shown, for example, that attention to market microstructure theory enables us to assess the likely validity of the independence assumption, to offer explanations of empirically observed cross-correlation patterns, to predict the existence of as-yet undiscovered patterns, and to make informed suggestions for improving volatility estimation methods. And conversely, of course, additional work along our lines may help promote the use of data in disciplining theory, by helping to sift the comparative merits of various competing theoretical microstructure models.

Novel uses of our results may also be possible. For example, the rate of decay of crosscorrelations might be used to assess the extent to which strategic traders are active in the market, and the sign and size of the contemporaneous correlation might be used to assess the degree of market maker risk aversion. Indeed market maker risk aversion might be time-varying, with associated time-varying cross-correlation structure between latent price and MSN. During crises, for example, market makers may be more risk averse, as borrowing and hedging possibilities are reduced. If so, the "normal pattern" of negative con-

\footnotetext{
${ }^{45}$ For an interesting related perspective, see Engle and Sun (2007). Their approach and environment (conditional duration modeling), however, are very different from ours.
} 
temporaneous cross-correlation and positive higher-order cross-correlations might switch to a "crisis pattern" of positive contemporaneous cross-correlation and negative higher-order cross-correlations. Such possibilities await future empirical exploration. 


\section{References}

Aghion, Philippe, Patrick Bolton, Christopher Harris, and Bruno Jullien (1991), "Optimal learning by experimentation," Review of Economic Studies, 58, 621-654. 12, 16, 47, 57

Aghion, Philippe, Maria Paz Espinosa, and Bruno Jullien (1993), "Dynamic duopoly with learning through market experimentation," Economic Theory, 3, 517-539. 12, 47

Ait-Sahalia, Yacine, P.A. Mykland, and L. Zhang (2010), "Ultra high frequency volatility estimation with dependent microstructure noise," Journal of Econometrics, forthcoming. 1

Andersen, Torben G., Tim Bollerslev, Peter F. Christoffersen, and Francis X. Diebold (2006), "Volatility and correlation forecasting," in "Handbook of Economic Forecasting," (edited by Elliot, Graham, Clive W. J. Granger, and Allan Timmermann), 777-878, Amsterdam: North-Holland. 1

Andersen, Torben G., Tim Bollerslev, and Francis X. Diebold (2010), "Parametric and nonparametric volatility measurement," in "Handbook of Financial Econometrics," (edited by Hansen, Lars P. and Yacine Aït-Sahalia), 67-137, Amsterdam: North-Holland. 1

Andersen, Torben G., Tim Bollerslev, Francis X. Diebold, and Heiko Ebens (2001a), "The distribution of realized stock return volatility," Journal of Financial Economics, 61, 4376. 1

Andersen, Torben G., Tim Bollerslev, Francis X. Diebold, and Paul Labys (2000), "Great realizations," Risk, 13, 105-108. 1

Andersen, Torben G., Tim Bollerslev, Francis X. Diebold, and Paul Labys (2001b), "The distribution of realized exchange rate volatility," Journal of the American Statistical Association, 96, 42-55. 1. 5

Andersen, Torben G., Tim Bollerslev, Francis X. Diebold, and Paul Labys (2003), "Modeling and forecasting realized volatility," Econometrica, 71, 579-625. 1

Bandi, Federico M. and Jeffrey R. Russell (2006), "Realized variance and market microstructure noise: Comment," Journal of Business and Economic Statistics, 24, 167-173. 20. 29

Bandi, Federico M. and Jeffrey R. Russell (2008), "Microstructure noise, realized variance, and optimal sampling," Review of Economic Studies, 75, 339-369. 1. 29 
Bandi, Federico M. and Jeffrey R. Russell (2010), "Market microstructure noise, integrated variance estimators, and the accuracy of asymptotic approximations," Journal of Econometrics, forthcoming. 32

Barndorff-Nielsen, Ole E., Peter R. Hansen, Asger Lunde, and Neil Shephard (2008a), "Designing realised kernels to measure the ex-post variation of equity prices in the presence of noise," Econometrica, 76, 1481-1536. 1]

Barndorff-Nielsen, Ole E., Peter R. Hansen, Asger Lunde, and Neil Shephard (2008b), "Multivariate realised kernels: Consistent positive semi-definite estimators of the covariation of equity prices with noise and non-synchronous trading," Manuscript, Oxford-Man Institute of Quantitative Finance, University of Oxford. 1

Barndorff-Nielsen, Ole E., Peter R. Hansen, Asger Lunde, and Neil Shephard (2009), "Realized kernels in practice: Trades and quotes," Econometrics Journal, 12, C1-C32. 35

Barndorff-Nielsen, Ole E., Peter R. Hansen, Asger Lunde, and Neil Shephard (2010), "Subsampling realised kernels," Journal of Econometrics, forthcoming. 1]

Barndorff-Nielsen, Ole E. and Neil Shephard (2002a), "Econometric analysis of realized volatility and its use in estimating stochastic volatility models," Journal of the Royal Statistical Society B, 64, 253-280. 1

Barndorff-Nielsen, Ole E. and Neil Shephard (2002b), "Estimating quadratic variation using realized variance," Journal of Applied Econometrics, 17, 457-477. 1

Barndorff-Nielsen, Ole E. and Neil Shephard (2007), "Variation, jumps, market frictions and high frequency data in financial econometrics," in "Advances in Economics and Econometrics, Theory and Applications, Ninth World Congress of Econometric Society," (edited by Blundell, Richard, Persson Torsten, and Whitney K. Newey), 328-372, Cambridge: Cambridge University Press. 1

Demsetz, Harold (1968), "The cost of transacting," Quarterly Journal of Economics, 82, 33-53. 24

Diamond, Douglas W. and Robert E. Verrecchia (1987), "Constraints on short-selling and asset price adjustment to private information," Journal of Financial Economics, 18, 277311. 14

Diebold, Francis X. (2006), "Realized variance and market microstructure noise: Comment," Journal of Business and Economic Statistics, 24, 181-183. 28, 29 
Easley, D., R.F. Engle, M. O'Hara, and L. Wu (2008), "Time-Varying Arrival Rates of Informed and Uninformed Trades," Journal of Financial Econometrics, 6, 171-207. 8

Easley, David and Maureen O'Hara (1987), "Price, trade size, and information in securities markets," Journal of Financial Economics, 19, 69-90. 17

Easley, David and Maureen O'Hara (1992), "Time and the process of security price adjustment," Journal of Finance, 47, 576-605. 2, 13, 14, 17, 26, 33, 44, 47, 48

Engle, Robert F. and Andrew J. Patton (2004), "Impacts of trades in an error-correction model of quote prices," Journal of Financial Markets, 7, 1-25. 9

Engle, Robert F. and Zheng Sun (2007), "When Is Noise Not Noise - A Microstructure Estimate of Realized Volatility," Manuscript, Stern School, New York University. 38

Fama, Eugene F. (1970), "Efficient Capital Markets: A review of theory and empirical work," Journal of Finance, 25, 383-417. 3

Foster, F. Douglas and S. Viswanathan (1996), "Strategic trading when agents forecast the forecasts of others," Journal of Finance, 51, 1437-1478. 37

Glosten, Lawrence R. and Lawrence E. Harris (1988), "Estimating the components of the bid/ask spread," Journal of Financial Economics, 21, 123-142. 37

Glosten, Lawrence R. and Paul R. Milgrom (1985), "Bid, ask, and transaction prices in a specialist market with heterogeneously informed traders," Journal of Financial Economics, 14, 71-100. 2, 13, 16

Hansen, Peter R., Jeremy Large, and Asger Lunde (2008), "Moving Average-Based Estimators of Integrated Variance," Econometric Reviews, 27, 79-111. 32, 33

Hansen, Peter R. and Asger Lunde (2006), "Realized variance and market microstructure noise," Journal of Business and Economic Statistics, 24, 127-161. 1, 6, 23, 24, 28, 29, 31, 32, 33, 34, 35, 37,

Hasbrouck, Joel (1993), "Assessing the quality of a security market: A new approach to transaction-cost measurement," Review of Financial Studies, 6, 191-212. 32

Hasbrouck, Joel (1996), "Modeling market microstructure time series," in "Statistical Methods in Finance," (edited by Maddala, Gangadharrao S. and Calyampudi R. Rao), 647-692, Amsterdam: North Holland. 37 
Hasbrouck, Joel (2002), "Stalking the efficient price in empirical microstructure specifications," Journal of Financial Markets, 5, 329-339. 2

Hasbrouck, Joel (2007), Empirical Market Microstructure, New York: Oxford University Press. 2, 25, 28

Kelly, David L. and Douglas G. Steigerwald (2004), "Private information and high-frequency stochastic volatility," Studies in Nonlinear Dynamics and Econometrics, 8, 1-28. 33, 44

Kyle, Albert S. (1985), "Continuous auctions and insider trading," Econometrica, 53, 13151336. 2, 17, 18, 27, 50, 51

Meddahi, Nour (2002), "A theoretical comparison between integrated and realized volatility," Journal of Applied Econometrics, 17, 479-508. 33

O'Hara, Maureen (1995), Market Microstructure Theory, Cambridge: Cambridge University Press. 2

Oomen, Roel C. A. (2006), "Realized variance and market microstructure noise: Comment," Journal of Business and Economic Statistics, 24, 195-202. 33

Owens, John and Douglas G. Steigerwald (2005), "Inferring information frequency and quality," Journal of Financial Econometrics, 3, 500-524. 9

Roll, Richard (1984), "A simple implicit measure of the effective bid-ask Spread in an efficient market," Journal of Finance, 39, 1127-1139. 2, 12, 25, 32

Zhang, Lan, Per A. Mykland, and Yacine Aït-Sahalia (2005), "A tale of two time scales: Determining integrated volatility with noisy high frequency data," Journal of the American Statistical Association, 100, 1394-1411. 1 


\section{Appendices}

\section{A Market Microstructure}

\section{A.1 Market Setup}

Let $\Omega_{t}$ denote all public information available at time $t$. The market maker in particular has no information beyond $\Omega_{t}{ }^{46}$ We analyze limit-order markets, populated by informed and uninformed traders. There are many market makers engaged in perfect competition and serving as counterparty to all trades. ${ }^{47}$ The timing of information and actions in any given period, $t$, which is infinitely often repeated, is as follows: ${ }^{48}$

1. $p_{t-1}^{*}$ becomes public information, thus $\left\{p_{t-1}^{*}\right\} \in \Omega_{t}$.

2. $p_{t}^{*}$ changes randomly.

3. The market maker observes $\Omega_{t}$ which contains at least all transaction prices and trades up to the previous period, i.e. $\left\{p_{i}, q_{i}\right\} \in \Omega_{t} \forall i<t$. $\Omega_{t}$ may contain additional information $\omega_{t}$ about the current strong form efficient price, $p_{t}^{*}$, for example the direction of the price innovation, $\left\{\operatorname{sgn}\left(\varepsilon_{t}\right)\right\}$. Conditional on this, the market maker forms his price expectations $p_{t}^{e}$.

4. The market maker quotes a pricing scheme for period $t$, i.e. a mid price $p_{t}^{\text {mid }}$ and a spread $2 s_{t} \geq 0$. The market maker is bound to transact one unit at this price.

5a. Informed traders are "active" with probability $\alpha$. An $\alpha<1$ can be interpreted as inattention of traders or as occasional absence of a signal about $p_{t}^{*}$. If they are active, they observe $p_{t}^{*}$ and the market maker pricing scheme $\left\{p_{t}^{\text {mid }}, s_{t}\right\}$. If based on their private knowledge $p_{t}^{*}>p_{t}^{a s k} \equiv p_{t}^{\text {mid }}+s_{t}$, then they try to buy an infinite amount, whereas if $p_{t}^{*}<p_{t}^{\text {bid }} \equiv p_{t}^{\text {mid }}-s_{t}$, they try to (short-) sell an infinite amount. However, the market maker fills the demand only up to his commitment limit, one unit. If a transaction takes place, the transaction price is $p_{t}=p_{t}^{a s k}$ or $p_{t}=p_{t}^{b i d}$, respectively. If

\footnotetext{
${ }^{46}$ Variance $\sigma^{2}$ and the probability density function of $\varepsilon_{t}$ are public knowledge. We assume perfect memory, $\Omega_{i} \subset \Omega_{t} \forall i \leq t$, and that given the information set $\Omega_{t}$ the market participants' optimizing behavior determines a unique market price $p\left(\Omega_{t}\right)$, with corresponding market return $\Delta p\left(\Omega_{t}, \Omega_{t-1}\right)$.

${ }^{47}$ At a minimum, we require one market maker and many potential competitors.

${ }^{48}$ The setup is similar to Easley and O'Hara (1992), where steps 5a and 5b are sequential, i.e. uninformed traders step in if the informed refuse to trade. A variant is the model of Kelly and Steigerwald (2004) with a random draw between steps $5 \mathrm{a}$ and $5 \mathrm{~b}$. That is, if the informed trader (5a) is drawn, and he refuses to trade, then the period ends without a trade.
} 
neither buy nor sell is profitable, or if informed traders are not active in this period, then no informed trade occurs.

5b. If there is no informed trade, uninformed traders trade instead randomly for exogenous reasons with probability $\beta$. If they trade, buying at $p_{t}^{a s k}$ and selling at $p_{t}^{b i d}$ has equal probability, which allows market makers to earn the spread without risk. The maker maker cannot distinguish informed from uninformed traders - the latter, however, are his only source of revenue.

6. If private information is valid for only one period, then the market continues with step 1. Otherwise, if information remains private for $T>1$ periods, no further information is revealed at this moment and the market continues with step 3. Eventually after $T$ loops $p_{t}^{*}$ becomes public information and the market continues with step 1 .

\section{A.2 Decision Problem of Market Maker}

Having detailed the market microstructure, we now describe the behavior of the market maker. The loss function of the market maker pins down the optimal spread size and the response to a trade, and is thus a key determinant of the sign of the cross-correlations. Before trading occurs, the market maker has a belief about $p_{t}^{*}$, summarized by the prior probability density function $f\left(p_{t}^{*}\right)$. We require $f\left(p_{t}^{*}\right)=f\left(p_{t-1}^{*}+\varepsilon_{t}\right)$ to be consistent with Assumption 2 and denote the corresponding cumulative distribution function with $F(\cdot)$. Let $\underline{p}$ and $\bar{p}$ denote the lower and upper end of the support of $f\left(p_{t}^{*}\right)$ that the market maker has determined by previous experimentation. ${ }^{49}$ We define the loss function of a market maker with risk aversion parameter $n \geq 1$ as $l_{n}(x)=-|x|^{n}$.

The market maker's loss in periods of informed trading is a function of the gap between the strong form efficient price and the transaction price. In periods without any informed trading the market maker suffers no loss, because any potential loss to a noise trade on the one side of the market is offset by an even larger (by $2 s$ ) gain from a noise trade on the other side of the market. The expected loss in period $t$ when the market price is set at $p_{t}$ is

$$
L_{n}\left(p_{t}, F(\cdot ; \underline{p}, \bar{p})\right)=-\int_{\underline{p}}^{\bar{p}}\left|\left(p_{t}-p_{t}^{*}\right) E\left(q_{t} \mid p_{t}^{*}, p_{t}, s_{t}\right)\right|^{n} f\left(p_{t}^{*}\right) d p_{t}^{*},
$$

where $E\left(q_{t} \mid p_{t}+s_{t}<p_{t}^{*}\right)=\alpha, E\left(q_{t} \mid p_{t}-s_{t}>p_{t}^{*}\right)=\alpha$, and $E\left(q_{t} \mid p_{t}-s_{t}<p_{t}^{*}<p_{t}+s_{t}\right)=$ $0 .{ }^{50}$ Throughout most of the paper, we consider the case of a risk-neutral market maker

\footnotetext{
${ }^{49}$ In the first period, either $\underline{p}$ and $\bar{p}$ are known, or are set to $\underline{p}=-\infty$ and $\bar{p}=\infty$.

${ }^{50} L_{n}\left(p_{t}, F(\cdot ; \underline{p}, \bar{p})\right)^{1 / n}$ is related to the $\ell_{n}$ metric. However, it differs in that it is reweighted, and sums over
} 
$(n=1)$. This assumption reflects that the market maker has a transaction limit of one unit per period. As long as one unit is small relative to market maker wealth, his risk aversion is negligible. An important special case for cross-correlations appears, however, in markets where one unit of $q$ is large relative to market maker wealth and thus risk aversion does matter. We will discuss the risk aversion case in section 3.3 .2 .

This setup has an immediate implication. If no informed traders are present in the market, then $E\left(q_{t} \varepsilon_{t-\tau}\right)=0 \forall t, \forall \tau$, because uninformed trades are unrelated to $p_{t}^{*}$. In contrast, for informed trades and sufficiently small risk aversion of the market maker $E\left(q_{t} \varepsilon_{t-\tau}\right) \geq 0$ $\forall t, \forall \tau \geq 0$, because informed traders buy only if the strong form price increased, and sell if it fell. Taken together it holds that ${ }^{51}$

$$
0 \leq E\left(q_{t} \varepsilon_{t-\tau}\right) \leq E\left(\left|\varepsilon_{t}\right|\right)<1, \forall t, \forall \tau
$$

The market maker wants to pick bid and ask prices that meet two competing objectives: on the one hand, minimizing current period losses to informed traders and simultaneously maximizing spread income from uninformed traders, and on the other hand, learning as much as possible about the latent price in order to provide a more accurate quote and thus incur smaller losses in future periods. Denoting the market maker's value function at time $t$ by $V_{t}\left(F_{t}\left(\cdot, \underline{p}_{t}, \bar{p}_{t}\right)\right)$ and his discount factor by $\delta$, the market maker solves the recursive problem

$$
\begin{aligned}
& V_{t}\left(F_{t}\left(\cdot ; \underline{p}_{t}, \bar{p}_{t}\right)\right)=\sum_{\omega_{t+1}} P\left(\omega_{t+1}\right)_{p^{b i d}, p^{a s k}}\left\{\alpha L_{n}\left(p^{b i d}, F_{t}\left(\cdot ; \underline{p}_{t}, p^{b i d}\right)\right)\right. \\
& +\delta V_{t+1}\left(\tilde{F}_{t+1} \mid q_{t}=-1\right)\left[\frac{(1-\alpha) \beta}{2}+\alpha\left(\frac{\beta}{2} F_{t}\left(p^{a s k}\right)+\left(1-\frac{\beta}{2}\right) F_{t}\left(p^{b i d}\right)-F_{t}\left(\underline{p}_{t}\right)\right)\right] \\
& +\frac{p^{a s k}-p^{b i d}}{2}\left[1-\alpha+\alpha\left(F_{t}\left(p^{a s k}\right)-F_{t}\left(p^{b i d}\right)\right)\right] \\
& +\delta V_{t+1}\left(\tilde{F}_{t+1} \mid q_{t}=+1\right)\left[\frac{(1-\alpha) \beta}{2}+\alpha\left(F_{t}\left(\bar{p}_{t}\right)-\left(1-\frac{\beta}{2}\right) F_{t}\left(p^{a s k}\right)-\frac{\beta}{2} F_{t}\left(p^{b i d}\right)\right)\right] \\
& \left.+\alpha L_{n}\left(p^{a s k}, F_{t}\left(\cdot ; p^{a s k}, \bar{p}_{t}\right)\right)\right\},
\end{aligned}
$$

where $\tilde{F}_{t+1}$ is the update of $F_{t}$ using information $\left\{\omega_{t+1}, q_{t}\right\}$, with $\bar{p}_{t+1}$ and $\underline{p}_{t+1}$ being the

infinitely many elements. In particular under zero spread and always active informed traders $\left(\left|E\left(q_{t} \mid p_{t}^{*}, p_{t}\right)\right|=\right.$ $\left.1 \forall p_{t} \neq p_{t}^{*}\right)$, we have for $n \rightarrow \infty$

$$
L_{n \rightarrow \infty}\left(p_{t}, F(\cdot ; \underline{p}, \bar{p})\right)^{1 / n}=-\sup \left\{\left|p_{t}-p_{t}^{*}\right|, p_{t}^{*} \in\{p \mid \underline{p} \leq p \leq \bar{p}, f(p)>0\}\right\}=-\sup \left\{\left|p_{t}-\bar{p}\right|,\left|p_{t}-\underline{p}\right|\right\} .
$$

${ }^{51}$ Note that $\left[E\left(\left|\varepsilon_{t}\right|\right)\right]^{2}<E\left(\left|\varepsilon_{t}\right|^{2}\right)=E\left(\varepsilon_{t}^{2}\right)=1$, where the first inequality follows from Jensen's inequality. Taking the square root we therefore get $E\left(\left|\varepsilon_{t}\right|\right)<1$. 
updated upper and lower bound of this distribution, and $P\left(\omega_{t+1}\right)$ is the probability that the market maker observes the signal $\omega_{t+1}$. The recursive problem (39) encompasses most cases that we discuss in this paper.

If $\omega_{t+1}$ contains only information about period $t$ and earlier, but no signal about $t+1$ values, and if the market maker takes the spread as given, ${ }^{52}$ then the market maker's only choice variable is the location of the spread interval, $p^{\text {mid }}$. Assuming that informed traders are active $(\alpha=1),(39)$ simplifies to

$$
\begin{aligned}
& V(F(; \underline{p}, \bar{p}))=\max _{p^{\text {mid }}} \quad\left[L _ { n } \left(p^{\text {mid }}-s, F\left(\cdot ; \underline{p}, p^{\text {mid }}-s\right)\right.\right. \\
& \quad+\delta V\left(\tilde{F}\left(\cdot ; \underline{p}, p^{\text {mid }}+s\right)\right)\left[\left(\frac{\beta}{2} F\left(p^{\text {mid }}+s\right)+\left(1-\frac{\beta}{2}\right) F\left(p^{\text {mid }}-s\right)-F(\underline{p})\right)\right] \\
& \quad+s \beta\left[F\left(p^{\text {mid }}+s\right)-F\left(p^{\text {mid }}-s\right)\right] \\
& \quad+\delta V\left(\tilde{F}\left(\cdot ; p^{\text {mid }}-s, \bar{p}\right)\left[\left(F(\bar{p})-\left(1-\frac{\beta}{2}\right) F\left(p^{\text {mid }}+s\right)-\frac{\beta}{2} F\left(p^{\text {mid }}-s\right)\right)\right]\right. \\
& \left.\quad+\quad L_{n}\left(p^{\text {mid }}+s, F\left(\cdot ; p^{\text {mid }}+s, \bar{p}\right)\right)\right] .
\end{aligned}
$$

Unfortunately, (39) and even 40 are hard to solve - in general the policy functions $p^{\text {bid }}(\cdot)$ and $p^{a s k}(\cdot)$ are not available in closed form. ${ }^{53}$

In the paper we look at specializations of the general market maker problem (39) and examine the effect of various model setups on the cross-correlation function. For both strong form and semi-strong form efficient returns we first examine the multiperiod case $(\delta>0)$, where private information is not revealed until after many periods. In subsections 3.3 and 4.3 we specialize to the one-period case $(\delta=0)$, a case where private information becomes public, and worthless, after only one period.

\section{B Proofs of Propositions}

\section{B.1 Proof of Proposition 1}

Proposition (Cross-correlations in the Easley-O'Hara model)

The contemporaneous cross-correlation in the Easley and O'Hara (1992) model is

$$
\operatorname{Corr}\left(\Delta p_{t}^{*}, \Delta u_{t}\right)=-\frac{1+e^{-r(T-1)}}{2 \sqrt{K}}<0,
$$

\footnotetext{
${ }^{52}$ The spread might be pinned down by competition (see Appendix E) or given exogenously by regulation.

${ }^{53}$ For characterizations of the general solution see Aghion et al. (1991) and Aghion et al. (1993). Their solution shows that in general optimal learning requires $\lambda_{t}$ in $(3)$ to vary over time.
} 
and the cross-correlations at sufficiently large nonzero displacements follow

$$
\begin{gathered}
\operatorname{Corr}\left(\Delta p_{t-\tau}^{*}, \Delta u_{t}\right)=\frac{e^{r}-1}{2 \sqrt{K}} e^{-r \tau}>0, \forall \tau \in[1, T-1] \\
\operatorname{Corr}\left(\Delta p_{t-T}^{*}, \Delta u_{t}\right)=\frac{e^{-r(T-1)}}{2 \sqrt{K}}>0
\end{gathered}
$$

where $K=K(r, T)$.

\section{Proof:}

Following the setup in Easley and O'Hara (1992), suppose the strong form efficient price process switches between a high state $\bar{p}$, a neutral state, and a low state $\underline{p}$, where $\chi$ is the probability of a non-neutral state and $\gamma$ the probability of a high state given that the state is non-neutral.

$$
p_{t}^{*}= \begin{cases}\bar{p} & \text { with probability } \chi \gamma \\ \gamma \underline{p}+(1-\gamma) \bar{p} & \text { w.p. } 1-\chi \\ \underline{p} & \text { w.p. } \chi(1-\gamma)\end{cases}
$$

Therefore, for $t=\kappa T, \kappa \in \mathbb{Z}$

$$
\Delta p_{t}^{*}= \begin{cases}\bar{p}-\underline{p} & \text { w.p. } \chi^{2} \gamma(1-\gamma) \\ \gamma(\bar{p}-\underline{p}) & \text { w.p. } 2 \chi(1-\chi)(1-\gamma) \\ 0 & \text { w.p. }(1-\chi)^{2}+\chi^{2}\left(\gamma^{2}+(1-\gamma)^{2}\right) \\ \gamma(\underline{p}-\bar{p}) & \text { w.p. } 2 \chi(1-\chi) \gamma \\ \underline{p}-\bar{p} & \text { w.p. } \chi^{2} \gamma(1-\gamma)\end{cases}
$$

and $\Delta p_{t}^{*}=0$ otherwise. Prices have the properties

$$
\begin{gathered}
E\left[\left(\Delta p_{t}^{*}\right)^{2}\right]=\frac{(\bar{p}-\underline{p})^{2}}{T}\left[2 \gamma^{2} \chi(1-\chi)(1-\gamma)+\chi^{2} \gamma(1-\gamma)+2 \gamma^{2} \chi(1-\chi) \gamma+\chi^{2} \gamma(1-\gamma)\right] \\
=\frac{(\bar{p}-\underline{p})^{2}}{T} 2 \chi \gamma(\gamma+\chi-2 \chi \gamma) \equiv \frac{\sigma^{2}}{T} \\
E\left(\Delta p_{t}^{*} \Delta p_{t}\right)=0 \\
E\left(\Delta p_{t-\tau}^{*} \Delta p_{t}^{*}\right)=0 .
\end{gathered}
$$

For ease of exposition let us focus on the case $\gamma=1 / 2$ and $\chi=1$, i.e. latent prices are high and low with equal probability. Using the result from Easley and O'Hara (1992) that 
transaction prices converge to the strong form efficient price at an exponential rate we get

$$
\begin{gathered}
\Delta p_{0}=\frac{\bar{p}-\underline{p}}{2}\left(e^{-r(T-1)}-1\right) \operatorname{sgn}\left(p_{-T}^{*}-\frac{\bar{p}+\underline{p}}{2}\right) \\
\Delta p_{\tau}=\frac{\bar{p}-\underline{p}}{2}\left(e^{-r(\tau-1)}-e^{-r \tau}\right) \operatorname{sgn}\left(p_{0}-\frac{\bar{p}+\underline{p}}{2}\right) \\
\Delta u_{0}=\frac{\bar{p}-\underline{p}}{2}\left(e^{-r(T-1)}-1\right) \operatorname{sgn}\left(p_{-T}^{*}-\frac{\bar{p}+\underline{p}}{2}\right)-\Delta p_{0}^{*} \\
\Delta u_{\tau}=\frac{\bar{p}-\underline{p}}{2}\left(e^{-r(\tau-1)}-e^{-r \tau}\right) \operatorname{sgn}\left(p_{0}-\frac{\bar{p}+\underline{p}}{2}\right)
\end{gathered}
$$

The contemporaneous cross-covariance $(\tau=0)$ is

$$
\begin{aligned}
\operatorname{Cov}\left(\Delta p_{t}^{*}, \Delta u_{t}\right) & =\frac{1}{T} E\left(\Delta p_{0}^{*} \Delta u_{0}\right) \\
& =-\frac{\sigma^{2}}{2 T}\left[1+e^{-r(T-1)}\right] .
\end{aligned}
$$

The second term inside the brackets is an artifact of $p_{t}^{*}$ not following a martingale. In the period of the efficient price change it is optimal for the market maker to set $p_{t}$ to the unconditional mean of $p_{t}^{*}$, thereby offsetting the effect of all previous learning, which the efficient price change rendered obsolete.

The cross-covariance for $\tau \in[1 ; T-1]$ is

$$
\begin{aligned}
\operatorname{Cov}\left(\Delta p_{t-\tau}^{*}, \Delta u_{t}\right) & =\frac{1}{T} E\left(\Delta p_{0}^{*} \Delta u_{\tau}\right) \\
& =\frac{\sigma^{2}}{2 T}\left(-e^{-r \tau}+e^{-r(\tau-1)}\right)
\end{aligned}
$$

and for $\tau=T$ we have

$$
\begin{aligned}
\operatorname{Cov}\left(\Delta p_{t-T}^{*}, \Delta u_{t}\right) & =\frac{1}{T} E\left(\Delta p_{-T}^{*} \Delta u_{0}\right) \\
& =\frac{\sigma^{2}}{2 T} e^{-r(T-1)}
\end{aligned}
$$


The variance of the noise is

$$
\begin{aligned}
\operatorname{Var}\left(\Delta u_{t}\right) & =\frac{1}{T}\left[\frac{(\bar{p}-\underline{p})^{2}}{T}\left(e^{-r(T-1)}-1\right)^{2}+\sigma^{2}+2 \frac{(\bar{p}-\underline{p})^{2}}{4}\left(e^{-r(T-1)}-1\right)\right. \\
& \left.+\sum_{\tau=1}^{T-1} \frac{(\bar{p}-\underline{p})^{2}}{4}\left(-e^{-r \tau}+e^{-r(\tau-1)}\right)^{2}\right] \\
& =\frac{\sigma^{2}}{T}\left[\frac{1}{2} e^{-2 r(T-1)}+\frac{1}{2}+\frac{1}{2}\left(-e^{r}+1\right)^{2} \frac{\left(e^{-2 r}\right)^{T-1}-1}{e^{-2 r}-1}\right] .
\end{aligned}
$$

Denoting the term in brackets by $K=K(r, T)$ we get for the contemporaneous crosscorrelation

$$
\operatorname{Corr}\left(\Delta p_{t}^{*}, \Delta u_{t}\right)=-\frac{1+e^{-r(T-1)}}{2 \sqrt{K}},
$$

for the cross-correlation at displacements $\tau \in[1 ; T-1]$

$$
\operatorname{Corr}\left(\Delta p_{t-\tau}^{*}, \Delta u_{t}\right)=\frac{-e^{-r \tau}+e^{-r(\tau-1)}}{2 \sqrt{K}},
$$

and for the cross-correlation at displacement $T$

$$
\operatorname{Corr}\left(\Delta p_{t-T}^{*}, \Delta u_{t}\right)=\frac{e^{-r(T-1)}}{2 \sqrt{K}} .
$$

Q.E.D.

\section{B.2 Proof of Proposition 2}

\section{Proposition (Cross-correlations in the Kyle model)}

The contemporaneous cross-correlation in Kyle (1985) is

$$
\operatorname{Corr}\left(\Delta p_{t}^{*}, \Delta u_{t}\right)=-\sqrt{\frac{T}{T^{2}+1}},
$$

the cross-correlations at displacements $\tau \in[1 ; T]$ are

$$
\operatorname{Corr}\left(\Delta p_{t-\tau}^{*}, \Delta u_{t}\right)=\sqrt{\frac{1}{T\left(T^{2}+1\right)}},
$$

and all higher order cross-correlations are zero.

\section{Proof:}

In order to present a closed-form solution we use continuous time, $t \in[0, T]$, but note 
that Kyle (1985) discussed the discrete time case as well. The discussion is based on the assumption of Kyle (1985) that the reaction functions for quantity demanded and prices are linear, i.e. that $\lambda_{t}=\lambda$, and $s_{t}=s$. Nonlinear solutions might nevertheless exist as well.

We assume semi-strong market efficiency, and so $s=\lambda$. We get from (16)

$$
\operatorname{Cov}\left(\Delta p_{t}^{*}, \Delta u_{t}\right)=-\frac{\sigma}{T}\left(\lambda E\left(q \varepsilon_{0}\right)-\sigma\right)<0
$$

From (17) the cross-covariance function at nonzero displacements

$$
\operatorname{Cov}\left(\Delta p_{t-\tau}^{*}, \Delta u_{t}\right)=\frac{\sigma}{T} \lambda E\left(q \varepsilon_{0}\right)>0
$$

is constant $\forall t \in[1, T-1]$, and zero $\forall t \geq T$.

More specifically, we derive based on (23) for the noise (assuming zero spread)

$$
\Delta u_{0}=\frac{\Delta p_{-T}^{*}}{T}-\int_{0}^{T-1} \frac{\sigma}{T-s} d B_{s}-\Delta p_{0}^{*}
$$

and for $\tau \in[1, T-1]$

$$
\Delta u_{\tau}=\frac{\Delta p_{0}^{*}}{T}+(T-\tau) \int_{\tau-1}^{\tau} \frac{\sigma}{T-s} d B_{s}-\int_{0}^{\tau-1} \frac{\sigma}{T-s} d B_{s} .
$$

The variance of the noise is therefore

$$
\begin{aligned}
\operatorname{Var}\left(\Delta u_{t}\right) & =\frac{1}{T}\left[E\left(\Delta u_{0}^{2}\right)+\sum_{t=1}^{T-1} E\left(\Delta u_{t}^{2}\right)\right] \\
& =\frac{\sigma^{2}}{T}\left[\frac{T+1}{T}+\frac{T-1}{T}+\frac{(T-1)^{2}}{T}\right] \\
& =\frac{\sigma^{2}}{T^{2}}\left(T^{2}+1\right) .
\end{aligned}
$$

The covariances are simply, at displacement zero

$$
\operatorname{Cov}\left(\Delta p_{t}^{*}, \Delta u_{t}\right)=\frac{1}{T} \operatorname{Cov}\left(\Delta p_{0}^{*},-\Delta p_{0}^{*}\right)=\frac{-\sigma^{2}}{T}
$$

and at higher order displacements

$$
\operatorname{Cov}\left(\Delta p_{t-\tau}^{*}, \Delta u_{t}\right)=\frac{1}{T} \operatorname{Cov}\left(\Delta p_{0}^{*}, \frac{\Delta p_{0}^{*}}{T}\right)=\frac{\sigma^{2}}{T^{2}}
$$

which leads directly to the cross-correlations given by Proposition 2, Q.E.D. 


\section{B.3 Proof of Proposition 3}

Proposition (Strong form cross-correlation, one period model)

$$
\begin{gathered}
\operatorname{Corr}\left(\Delta p_{t}^{*}, \Delta u_{t}\right)=\frac{1}{\sqrt{2}} \frac{s E\left(q_{t} \varepsilon_{t}\right)-\sigma}{\sqrt{\phi s^{2}+\sigma^{2}-2 s \sigma E\left(q_{t} \varepsilon_{t}\right)}} \\
\operatorname{Corr}\left(\Delta p_{t-1}^{*}, \Delta u_{t}\right)=-\operatorname{Corr}\left(\Delta p_{t}^{*}, \Delta u_{t}\right)
\end{gathered}
$$

\section{Proof:}

With $T=1$, no extra information, $\lambda_{t}=\lambda, s_{t}=s$, and thus $\phi_{t}=\phi \forall t$, the variance term (21) simplifies to $\operatorname{Var}\left(\Delta u_{t}\right)=2\left(\sigma^{2}+\phi s^{2}\right)-4 s \sigma E\left(q_{t} \varepsilon_{t}\right)$. Plugging this into 222) gives the desired result. Q.E.D.

\section{B.4 Proof of Proposition 4}

\section{Proposition (Bounds of contemporaneous cross-correlation)}

$$
-\frac{1}{\sqrt{2}} \leq \operatorname{Corr}\left(\Delta p_{t}^{*}, \Delta u_{t}\right) \leq 0
$$

\section{Proof:}

Negativity can be seen as follows. Uninformed traders trade randomly $\left(E\left(q_{t} \mid \varepsilon_{t}\right)=0\right)$, thus for them we have $s E\left(q_{t}^{u} \varepsilon_{t}\right)=0$. In contrast, informed traders buy $\left(q_{t}=+1\right)$ only when $\sigma \varepsilon_{t}>s$ and sell $\left(q_{t}=-1\right)$ only when $\sigma \varepsilon_{t}<-s$. Thus in a market of only informed traders $\sigma q_{t}^{i} \varepsilon_{t}>s \geq 0 \forall t$. Therefore we can write

$$
1=E\left(q_{t}^{i 2} \varepsilon_{t}^{2}\right)>E\left(\frac{s}{\sigma} q_{t}^{i} \varepsilon_{t}\right)>E\left(\frac{s^{2}}{\sigma^{2}}\right)>0
$$

so in particular $\sigma>s E\left(q_{t}^{i} \varepsilon_{t}\right)>0$. Combining informed and uninformed trades we have

$$
\sigma \geq s E\left(q_{t} \varepsilon_{t}\right)>0
$$

which implies that the contemporaneous cross-correlation (24) is negative.

Further, 24 is bounded from below by $-1 / \sqrt{2}$, which we prove by contradiction. Suppose this was not the case, then from (24)

$$
s E\left(q_{t} \varepsilon_{t}\right)-\sigma<-\sqrt{\phi s^{2}+\sigma^{2}-2 s \sigma E\left(q_{t} \varepsilon_{t}\right)} .
$$


Squaring both sides and simplifying gives the condition

$$
\left[E\left(q_{t} \varepsilon_{t}\right)\right]^{2}>\phi
$$

but by Jensen's inequality and $\beta=1$

$$
\left[E\left(q_{t} \varepsilon_{t}\right)\right]^{2} \leq E\left(q_{t}^{2} \varepsilon_{t}^{2}\right)=1
$$

which contradicts 41). Q.E.D.

\section{B.5 Proof of Proposition 5}

\section{Proposition (Optimal Mid Price)}

$$
\begin{aligned}
p(1) & =\operatorname{Median}\left(p_{t}^{*}\right) \\
p(2) & =\mathrm{E}\left(p_{t}^{*}\right) \\
p(\infty) & =\operatorname{Midsupport}\left(p_{t}^{*}\right)
\end{aligned}
$$

\section{Proof:}

The first two equations in the proposition ${ }^{54}$ are the well-known result that the median is the best predictor under linear (absolute) loss, whereas the mean is the best predictor under squared loss. The third equation is obtained by first noting that for any density $f(\cdot)$, which has all moments, we can apply Leibnitz's rule. Thus we obtain for (26) the first order condition

$$
\int_{\underline{p}}^{p(n)}\left(p(n)-p^{*}\right)^{n-1} f\left(p^{*}\right) d p^{*}-\int_{p(n)}^{\bar{p}}\left(p^{*}-p(n)\right)^{n-1} f\left(p^{*}\right) d p^{*}=0 .
$$

Rewriting (42) as a metric

$$
\begin{aligned}
\lim _{n \rightarrow \infty} & \left(\int_{\underline{p}}^{p(n)}\left(p(n)-p^{*}\right)^{n-1} f\left(p^{*}\right) d p^{*}\right)^{1 /(n-1)} \\
= & \lim _{n \rightarrow \infty}\left(\int_{p(n)}^{\bar{p}}\left(p^{*}-p(n)\right)^{n-1} f\left(p^{*}\right) d p^{*}\right)^{1 /(n-1)}
\end{aligned}
$$

\footnotetext{
${ }^{54}$ We assume $n \geq 1$ throughout, because this implies realistic market maker preferences. However, (26) can be solved for any $n \geq 0$. In particular, $p^{\text {mid }}(0)$ is the mode of $f(\cdot)$ when $s=0$, or the highest density (connected) region when $s>0$. For $n \notin\{1,2, \infty\}$ no explicit solution exists, and for $n>25$ even obtaining numerical solutions creates difficulty for non-trivial distribution functions $f(\cdot)$.
} 
which after taking the limit degenerates to the sup norm

$$
\sup _{p^{*} \in[\underline{p}, p(\infty)]}\left(p(\infty)-p^{*}\right)=\sup _{p^{*} \in[p(\infty), \bar{p}]}\left(p^{*}-p(\infty)\right)
$$

gives

$$
p(\infty)=\frac{p+\bar{p}}{2}
$$

Thus, by monotonicity (43) solves 42 for $n \rightarrow \infty$. Q.E.D.

\section{B.6 Proof of Proposition 6}

\section{Proposition (Cross-correlation under market maker information)}

If $\Omega_{t}=\left\{\operatorname{sgn}\left(\varepsilon_{t}\right), p_{t-1}^{*}\right\}$ and Assumption 2 holds, then the optimal market maker response $R\left(\left\{\operatorname{sgn}\left(\varepsilon_{t}\right)\right\}\right)$ strictly increases in risk aversion, $n \geq 1$, without bound. If, further, the distribution of the expected latent price with support $\left[\underline{p}^{*}, \bar{p}^{*}\right]$ satisfies

$$
\left[\frac{\underline{p}_{t}^{*}+\bar{p}_{t}^{*}}{2}-p_{t-1}^{*}\right] \operatorname{sgn}\left(\varepsilon_{t}\right)>s+\frac{\sigma}{E\left(\left|\varepsilon_{t}\right|\right)},
$$

then $\exists n_{0}>1$ such that $\forall n>n_{0}$ it holds that $\operatorname{Corr}\left(\Delta p_{t}^{*}, \Delta u_{t}\right)>0$.

\section{Proof:}

The new information $\omega_{t}$ is now replaced by two parts: The first part reflects as before information about $p_{t-1}^{*}$, i.e. $\omega_{t}=p_{t-1}^{*}-p_{t-1}+\left(s_{t-1}-\lambda_{t-1}\right) q_{t-1}$. The second, and new, part reflects the extra information about $\Delta p_{t}^{*}$, and the response of the market maker to it. To be specific, we assume that this extra information is the direction of the latent price change $\left\{\operatorname{sgn}\left(\varepsilon_{t}\right)\right\}$. If the distribution of expected latent price changes at the beginning of each period is the same, we can write the market maker response to this extra information as $R\left(\operatorname{sgn}\left(\varepsilon_{t}\right)\right)=R \operatorname{sgn}\left(\varepsilon_{t}\right)$. From $\Delta p_{t}=-p_{t-1}+p_{t-1}^{*}+s q_{t}+R(\cdot)$, and

$$
\begin{aligned}
E\left(\Delta p_{t} \varepsilon_{t}\right) & =E\left[\left(-p_{t-1}+p_{t-1}^{*}+s q_{t}+R(\cdot)\right) \varepsilon_{t}\right] \\
& =\frac{1}{2} E\left[\left(s q_{t}+R(\cdot)\right) \varepsilon_{t} \mid \varepsilon_{t}>0\right]+\frac{1}{2} E\left[\left(s q_{t}-R(\cdot)\right) \varepsilon_{t} \mid \varepsilon_{t}<0\right] \\
& =R E\left(\left|\varepsilon_{t}\right|\right)+s E\left(q_{t} \varepsilon_{t}\right) .
\end{aligned}
$$

Plugging (44) with $E\left(\Delta p_{t} \Delta p_{t}^{*}\right)=\sigma E\left(\Delta p_{t} \varepsilon_{t}\right)$ into (9) implies that the contemporaneous cross-covariance is positive if and only if

$$
R>\frac{\sigma-s E\left(q_{t} \varepsilon_{t}\right)}{E\left(\left|\varepsilon_{t}\right|\right)}
$$


Because $E\left(q_{t} \varepsilon_{t}\right)>-E\left(\left|\varepsilon_{t}\right|\right)$ we have as sufficient condition

$$
R>s+\frac{\sigma}{E\left(\left|\varepsilon_{t}\right|\right)}
$$

To satisfy 46) we need for $p_{t}^{e}=p_{t-1}^{*}+R(\cdot)=p_{t-1}^{*}+R \operatorname{sgn}\left(\varepsilon_{t}\right)$ that

$$
p_{t}^{e} \begin{cases}>p_{t-1}^{*}+s+\frac{\sigma}{E\left(\left|\varepsilon_{t}\right|\right)} & \text { for } \varepsilon>0 \\ <p_{t-1}^{*}-s-\frac{\sigma}{E\left(\left|\varepsilon_{t}\right|\right)} & \text { for } \varepsilon<0\end{cases}
$$

From Proposition 5 for any $p(n) \in\left[\operatorname{Median}(p) ; \frac{p+\bar{p}}{2}\right]$ there is a risk aversion level $n$ such that market makers will - after observing the signal $\left\{\operatorname{sgn}\left(\varepsilon_{t}\right)\right\}$ - quote this price as mid price $p_{t}^{e}$. Therefore, for all distributions $f\left(p^{*}\right)$ which satisfy (27), a sufficiently large $n$ leads to a market maker response which satisfies (45) and thus to a positive contemporaneous cross-covariance. Q.E.D.

\section{B.7 Proof of Proposition 7}

Proposition (Semi-strong form cross-correlation, one period model)

The contemporaneous cross-correlation is

$$
\operatorname{Corr}\left(\Delta \tilde{p}_{t}^{e}, \Delta u_{t}\right)=\frac{2 \phi \lambda-\sigma E\left(q_{t} \varepsilon_{t}\right)}{\sqrt{\sigma^{2}-2 \sigma \lambda E\left(q_{t} \varepsilon_{t}\right)+2 \phi \lambda^{2}}} \frac{\operatorname{sgn}(s-\lambda)}{\sqrt{2 \phi}}
$$

The cross-correlation at displacement one equals

$$
\operatorname{Corr}\left(\Delta \tilde{p}_{t-1}^{e}, \Delta u_{t}\right)=\frac{-\phi \lambda}{\sqrt{\sigma^{2}-2 \sigma \lambda E\left(q_{t} \varepsilon_{t}\right)+2 \lambda^{2}}} \frac{\operatorname{sgn}(s-\lambda)}{\sqrt{2 \phi}}
$$

All cross-correlations at higher displacements are zero.

\section{Proof:}

The expressions for the cross-correlations follow directly from their multiperiod counterparts. In the setup of section 4.1 the semi-strong form efficient price has the unconditional 
variance (36) and the corresponding noise has an unconditional variance of

$$
\begin{aligned}
\operatorname{Var}\left(\Delta u_{t}\right) & =\frac{1}{T} \sum_{t=0}^{T-1} \operatorname{Var}\left(\Delta u_{t}\right) \\
& =\frac{1}{T}\left\{\sum_{i=0}^{T-1}\left[\phi_{i}\left(\lambda_{i}-s_{i}\right)^{2}+\phi_{i-1}\left(\lambda_{i-1}-s_{i-1}\right)^{2}\right]\right. \\
& \left.-2 \sum_{i=1}^{T-1} E\left(q_{t} q_{t-1}\right)\left(\lambda_{i}-s_{i}\right)\left(\lambda_{i-1}-s_{i-1}\right)\right\} .
\end{aligned}
$$

The contemporaneous cross-correlation is

$$
\operatorname{Corr}\left(\Delta \tilde{p}_{t}^{e}, \Delta u_{t}\right)=\frac{\operatorname{Cov}\left(\Delta \tilde{p}_{t}, \Delta u_{t}\right)}{\sqrt{\operatorname{Var}\left(\Delta \tilde{p}_{t}^{e}\right) \operatorname{Var}\left(\Delta u_{t}\right)}}
$$

where $\operatorname{Cov}\left(\Delta \tilde{p}_{t}^{e}, \Delta u_{t}\right)$ is given by (29). All other cross-correlation can be obtained analogously.

For $T=1$, spread and adverse selection parameter are constants, i.e. $s_{t}=s$ and $\lambda_{t}=\lambda$ $\forall t$, and the variance terms (36) and 47) simplify radically to

$$
\begin{gathered}
\operatorname{Var}\left(\Delta \tilde{p}_{t}^{e}\right)=\sigma^{2}-2 \sigma \lambda E\left(q_{t} \varepsilon_{t}\right)+2 \phi \lambda^{2}, \\
\operatorname{Var}\left(\Delta u_{t}\right)=2 \phi(s-\lambda)^{2},
\end{gathered}
$$

where we have used that $q_{t}$ is serially uncorrelated. Finally, from $(29)$ and (31), we get for $T=1$ the covariances

$$
\operatorname{Cov}\left(\Delta \tilde{p}_{t}^{e}, \Delta u_{t}\right)=(s-\lambda)\left[2 \phi \lambda-\sigma E\left(q_{t} \varepsilon_{t}\right)\right]
$$

and

$$
\operatorname{Cov}\left(\Delta \tilde{p}_{t-1}^{e}, \Delta u_{t}\right)=\phi \lambda(\lambda-s)
$$

Combining these with the variances immediately gives the cross-correlations stated in proposition 7. Q.E.D.

\section{Example of Optimal Learning}

Here we show that under signal certainty, the cross-correlation at nonzero displacements falls with $\tau$. Consider a signal that is known to be free of noise and strategic manipulation by market participants. To learn as much as possible the market maker minimizes the length of 
the interval in which $p_{t}^{*}$ may be located. Even with a uniform prior belief, $F$, the posterior $\tilde{F}$ is not uniform (as in Aghion et al. (1991)), but step-uniform, because of the noise induced by uninformed trading. For the upper and lower end of the distribution to be a sufficient statistic for market maker believes, we have to additionally assume the absence of noise traders $(\beta=0)$. With these assumptions 400 collapses to

$$
\begin{aligned}
V(\underline{p}, \bar{p}) & =\max _{p^{m i d}}-\int_{\underline{p}}^{p^{\text {mid }}-s} \frac{\left|p^{\text {mid }}-s-p^{*}\right|}{p^{\text {mid }}-s-\underline{p}} d p^{*}+\delta V\left(\underline{p}, p^{\text {mid }}-s\right)\left(p^{\text {mid }}-s-\underline{p}\right) \\
& +\delta V\left(p^{\text {mid }}+s, \bar{p}\right)\left(\bar{p}-p^{\text {mid }}-s\right)-\int_{p^{\text {mid }}+s}^{\bar{p}} \frac{\left|p^{*}-p^{\text {mid }}-s\right|}{\bar{p}-p^{\text {mid }}-s} d p^{*} .
\end{aligned}
$$

A solution to this example is the market maker to do repeated bisections, setting $p_{t}^{\text {mid }}=$ $\frac{\bar{p}_{t}+\underline{p}_{t}}{2}$ in every period. To see this, let us assume risk neutrality $(n=1)$, always active informed traders, and no uninformed trades (for which a zero spread is sufficient, but not necessary) which simplifies this recursive problem to

$$
\begin{aligned}
V(\underline{p}, \bar{p}) & =\max _{p}\left[-\int_{\underline{p}}^{p-s}\left(p-s-p^{*}\right) f\left(p^{*}\right) d p^{*}+V(\underline{p}, p-s) F(p-s)\right. \\
& +V(p-s, p+s)(F(p+s)-F(p-s))+V(p+s, \bar{p})(1-F(p)) \\
& \left.-\int_{p+s}^{\bar{p}}\left(p^{*}-p-s\right) f\left(p^{*}\right) d p^{*}\right] .
\end{aligned}
$$

To simplify the problem further, we assume as in the example in Aghion et al. (1991) that $f(\cdot)$ is uniform. Then, in fact, the location of the interval $[\underline{p}, \bar{p}]$ does not matter for describing information content. The length of it alone, $m=\bar{p}-\underline{p}$, summarizes the state of learning. (48) becomes with $p=\alpha m+\underline{p}$

$$
\begin{aligned}
V(m) & =\max _{\alpha} \quad-\frac{1}{2 m}(\alpha m)^{2}+\delta V(\alpha m) \alpha \\
& -\frac{1}{2 m}((1-\alpha) m)^{2}+\delta V((1-\alpha) m)(1-\alpha)+V(2 s) \frac{2 s}{m} \\
& =\max _{\alpha} \quad \delta V(\alpha m) \alpha+\delta V((1-\alpha) m)(1-\alpha)-\frac{1}{2 m}\left((\alpha m-s)^{2}+((1-\alpha) m-s)^{2}\right) \\
& =\max _{\alpha} \quad \delta V(\alpha m) \alpha+\delta V((1-\alpha) m)(1-\alpha) \\
& -\frac{1}{2 m}\left(2 \alpha^{2} m^{2}+2 s^{2}+m^{2}-2 \alpha m^{2}-2 m s\right) .
\end{aligned}
$$

From the first order condition for the maximum we get $\alpha=1 / 2$, thus optimal learning is achieved by repeated bisections.

This result is driven by uniformity and the zero spread, which ensures that Assumption 
2 holds in every period, in particular that $f(\cdot)$ in $(48)$ is always symmetric. Thereby, the term in brackets in 48) is symmetric around the symmetry point of $f(\cdot)$ as well, and the optimal $p^{\text {mid }}$ equals the median, and the midpoint of the support of $f(\cdot)$. For non-uniform $f(\cdot)$ the solution path over time is specific to the shape of $f(\cdot)$ and has to be determined numerically.

Therefore, the market maker's optimal pricing rule follows repeated bisections, i.e. he sets

$p_{t}^{\text {mid }}=\frac{\bar{p}_{t}+\underline{p}_{t}}{2}$ in every period. Therefore $p_{t}^{\text {mid }}$ converges, in smaller and smaller steps, towards $p^{*}$, and the probability of $p^{*} \in\left[p^{m i d}-s, p^{m i d}+s\right]$ converges to unity. The smaller the interval $[\underline{p}, \bar{p}]$ of possible $p^{*}$ becomes, the less likely will the choice of $p^{\text {mid }}$ allow profitable informed trades. Therefore $E\left(q_{0} \varepsilon_{0}\right)$ decreases over time. Likewise, as more and more information is already contained in $[\underline{p}, \bar{p}], E\left(\omega_{t} \varepsilon_{0}\right)$ decreases as well. Overall, the cross-correlation at nonzero displacements falls with $\tau$.

\section{Effect of Risk Aversion on Optimal Price}

Here we show that high risk aversion pushes the optimal price toward the midpoint of the support. In other words, if $f(\cdot)$ is without loss of generality right-skewed, then $p(n)$ is increasing in $n, \forall n \geq 1$. First, note that $p(n), p(n) \in[\underline{p}, \bar{p}],{ }^{55}$ is continuous. If $\underline{p}$ or $\bar{p}$ are infinite, we replace these bounds with a function of $n$, thereby making the domain of $p$ compact. As $f(\cdot)$ and all components of the integral are continuous functions, the theorem of the maximum gives continuity of $p(n)$.

Next, to evaluate how the optimal price $p(n)$ responds to changes in risk aversion $n$, take the total differential of (42) and rearrange to obtain

$$
\begin{aligned}
& \frac{d p(n)}{d n}=\frac{1}{n-1} \times\left\{-\int_{\underline{p}}^{p(n)}\left(p(n)-p^{*}\right)^{n-1} \ln \left(p(n)-p^{*}\right) f\left(p^{*}\right) d p^{*}\right. \\
& \left.+\int_{p(n)}^{\bar{p}}\left(p^{*}-p(n)\right)^{n-1} \ln \left(p^{*}-p(n)\right) f\left(p^{*}\right) d p^{*}\right\} / \\
& \quad\left\{\int_{\underline{p}}^{p(n)}\left(p(n)-p^{*}\right)^{n-2} f\left(p^{*}\right) d p^{*}+\int_{p(n)}^{\bar{p}}\left(p^{*}-p(n)\right)^{n-2} f\left(p^{*}\right) d p^{*}\right\}
\end{aligned}
$$

In the following argument we use that $f(\cdot)$ is monotone and assume without loss of

\footnotetext{
${ }^{55}$ We suppress the asterisk from $\underline{p}^{*}$ and $\bar{p}^{*}$ and replace $p_{t}^{\text {mid }}(n)$ by $p(n)$ to simplify notation.
} 
generality that $f(\cdot)$ is monotonically decreasing. This means $f(\cdot)$ is right-skewed on $[\underline{p}, \bar{p}]$, which occurs if the market maker has some information that the strong form efficient price has increased. Under this assumption (49) is positive. To see this, note first that both terms in the denominator are positive. To economize notation we replace $p \equiv p(n), d \equiv p(n)-\underline{p}$ and $x \equiv p^{*}$. The numerator can be broken up into three parts:

$$
\begin{aligned}
& -\int_{\underline{p}}^{p}(p-x)^{n-1} \ln (p-x) f(x) d x+\int_{p}^{\bar{p}}(x-p)^{n-1} \ln (x-p) f(x) d x \\
& =-\int_{p-d}^{p-1}(p-x)^{n-1} \ln (p-x) f(x) d x+\int_{p+1}^{p+d}(x-p)^{n-1} \ln (x-p) f(x) d x \\
& -\int_{p-1}^{p}(p-x)^{n-1} \ln (p-x) f(x) d x+\int_{p}^{p+1}(x-p)^{n-1} \ln (x-p) f(x) d x \\
& +\int_{p+d}^{p}(x-p)^{n-1} \ln (x-p) f(x) d x .
\end{aligned}
$$

The first term, which exists only for $d>1$, gives

$$
\begin{aligned}
& \int_{p-d}^{p-1}(p-x)^{n-1} \ln (p-x) f(x) d x+\int_{p+1}^{p+d}(x-p)^{n-1} \ln (x-p) f(x) d x \\
= & -\int_{p+1}^{p+d}(x-p)^{n-1} \ln (x-p) f(2 p-x) d x \\
& +\int_{p+1}^{p+d}(x-p)^{n-1} \ln (x-p) f(x) d x \\
= & \int_{p+1}^{p+d}(x-p)^{n-1} \ln (x-p)[-f(2 p-x)+f(x)] d x \\
\geq & \int_{p+1}^{p+d}(x-p)^{n-1} \ln (d)[-f(2 p-x)+f(x)] d x \\
= & -\int_{p-d}^{p-1}(p-x)^{n-1} \ln (d) f(x) d x+\int_{p+1}^{p+d}(x-p)^{n-1} \ln (d) f(x) d x .
\end{aligned}
$$


The second term is for $d \geq 1$

$$
\begin{aligned}
& \int_{p-1}^{p}(p-x)^{n-1} \ln (p-x) f(x) d x+\int_{p}^{p+1}(x-p)^{n-1} \ln (x-p) f(x) d x \\
= & -\int_{p}^{p+1}(x-p)^{n-1} \ln (x-p) f(2 p-x) d x \\
& +\int_{p}^{p+1}(x-p)^{n-1} \ln (x-p) f(x) d x \\
= & \int_{p}^{p+1}(x-p)^{n-1} \ln (x-p)[f(x)-f(2 p-x)] d x \geq 0 .
\end{aligned}
$$

For $d<1$ the last inequality of the calculations for the second term is instead

$$
\begin{aligned}
& \int_{p}^{p+1}(x-p)^{n-1} \ln (x-p)[f(x)-f(2 p-x)] d x \\
\geq & \int_{p}^{p+d}(x-p)^{n-1}[f(x)-f(2 p-x)] d x \ln (d) \geq 0 .
\end{aligned}
$$

And for the last term we can write

$$
-\int_{p+d}^{\bar{p}}(x-p)^{n-1} \ln (x-p) f(x) d x>-\int_{p+d}^{\bar{p}}(x-p)^{n-1} \ln (d) f(x) d x .
$$

Using (51), (52), and (54), 50) becomes

$$
50]>\left[-\int_{p-d}^{p-1}(p-x)^{n-1} f(x) d x+\int_{p+1}^{p}(x-p)^{n-1} f(x) d x\right] \ln (d)
$$




$$
\begin{aligned}
& >\left[-\int_{p-d}^{p-1}(p-x)^{n-1} f(x) d x-\int_{p-1}^{p}(p-x)^{n-1} f(x) d x\right. \\
& \left.+\int_{p}^{p+1}(x-p)^{n-1} f(x) d x+\int_{p+1}^{\bar{p}}(x-p)^{n-1} f(x) d x\right] \ln (d) \\
& =\left[-\int_{p-d}^{p}(p-x)^{n-1} f(x) d x+\int_{p}^{\bar{p}}(x-p)^{n-1} f(x) d x\right] \ln (d) \\
& =0,
\end{aligned}
$$

where the inequality follows from the monotonicity of $f(\cdot)$, and the last equality follows from the first order condition 42.

Likewise, for $d<1$, using (53) we have

$$
\begin{aligned}
550) & >\left[-\int_{p-d}^{p}(p-x)^{n-1} f(x) d x+\int_{p}^{\underline{p}}(x-p)^{n-1} f(x) d x\right] \ln (d) \\
& =0 .
\end{aligned}
$$

Therefore the numerator is positive and

$$
\frac{d p(n)}{d n}>0
$$

for right-skewed distributions. Combining this with the fact that $p(1)=\operatorname{Median}\left(p^{*}\right)$ and $p(\infty)=\operatorname{Midsupport}\left(p^{*}\right)$ we conclude that $p(n)$ monotonically increases from the median to the midpoint of the support of the efficient price distribution $f(\cdot)$, if $f(\cdot)$ is right-skewed. Analogously, for left-skewed $f(\cdot), p(n)$ monotonically decreases from the median to the midpoint of the support.

\section{E Zero Profit Condition Under Perfect Competition}

In the following example we show that $s$ is uniquely determined as a competitive outcome. Under perfect competition the zero profit condition requires the market maker's losses in trades with informed traders to exactly offset the spread earned from trades with uninformed traders. An increase in the spread benefits the market maker in three ways: it increases his spread income from uninformed traders, makes uninformed trades more likely, and reduces 
his losses to informed traders. This can be written as

$$
\begin{gathered}
-\int_{-\infty}^{p^{b i d}}\left(p^{b i d}-p^{*}\right)^{n} f\left(p^{*}\right) d p^{*}-\int_{p^{a s k}}^{\infty}\left(p^{*}-p^{a s k}\right)^{n} f\left(p^{*}\right) d p^{*} \\
+\left(\int_{p^{b i d}}^{p^{a s k}} f\left(p^{*}\right) d p^{*}\right) \frac{p^{a s k}-p^{b i d}}{2}=0 .
\end{gathered}
$$

Clearly, as in section 3.3 .2 the bid and ask price grow in $n$, without bound as $n \rightarrow \infty$ if $f(\cdot)$ has unbounded support.

For simplicity, we now assume risk neutrality $(n=1)$ in a one-period model $(\delta=0)$, where informed traders are always active. Using the symmetry of the expected density $f\left(p^{*}\right)$ around $p^{\text {mid }}, 55$ becomes a problem of setting $p^{\text {mid }}$ and $s$.

$$
-\int_{-\infty}^{p^{m i d}-s}\left(p^{*}-p^{m i d}+s\right) f\left(p^{*}\right) d p^{*}=\left(\int_{p^{m i d}-s}^{p^{m i d}} f\left(p^{*}\right) d p^{*}\right) s .
$$

Then, with $F(\cdot)$ denoting the cumulative density function of $f(\cdot)$ (whose expected value is assumed to exist)

$$
\begin{gathered}
-\left(E\left(p^{*}\right)||_{-\infty}^{m^{m i d}-s} \cdot F\left(p^{m i d}-s\right)-\left(p^{m i d}-s\right) F\left(p^{m i d}-s\right)\right) \\
=\left(F\left(p^{m i d}\right)-F\left(p^{m i d}-s\right)\right) s,
\end{gathered}
$$

where $\left.E\left(p^{*}\right)\right|_{-\infty} ^{p}$ denotes the expectation of $p^{*}$ over the distribution $f\left(p^{*}\right)$ restricted to the interval $[-\infty, p] . p^{\text {mid }}$ is given by the optimal learning rule. (57) is one equation in the one unknown, $s$.

$$
p^{\text {mid }}-\left.E\left(p^{*}\right)\right|_{-\infty} ^{p^{m i d}-s}=\frac{s F\left(p^{m i d}\right)}{F\left(p^{m i d}-s\right)}
$$

The left-hand side (LHS) is monotonically increasing in $s$ from some positive number to positive infinity. The right-hand side (RHS) is monotonically increasing in $s$, from 0 to positive infinity. It can be shown that the RHS increases faster than the LHS and that this difference in slope does not go to zero as $s$ becomes larger. Differentiating (56) using Leibnitz's rule, we get

$$
\begin{gathered}
-\left(p^{\text {mid }}-s-p^{m i d}+s\right) f\left(p^{m i d}-s\right)\left(\frac{-1}{2}\right)-\int_{-\infty}^{p^{m i d}-s} \frac{1}{2} f\left(p^{*}\right) d p^{*} \\
<-f\left(p^{m i d}-s\right)\left(\frac{-1}{2}\right) s+\frac{1}{2}\left(\int_{p^{m i d}-s}^{p^{m i d}} f\left(p^{*}\right) d p^{*}\right)
\end{gathered}
$$


and

$$
-\frac{1}{2} F\left(p^{m i d}-s\right)<\frac{s}{2} f\left(p^{m i d}-s\right)+\frac{1}{2}\left(F\left(p^{m i d}\right)-F\left(p^{m i d}-s\right)\right) .
$$

Therefore

$$
0<s f\left(p^{m i d}-s\right)+F\left(p^{m i d}\right)
$$

which holds always by definition. This shows two things: Firstly, the RHS in (56) is increasing faster than the LHS. And secondly, the difference in slope is always at least $F\left(p^{\text {mid }}\right)>0$. Hence we have proven that there is a single crossing and $s$ is determined uniquely.

If the LHS in (58) is very small because the support of the distribution became very small by learning, then $s$ must be small as well. Hence, as market makers learn, the spread $s$ in the market shrinks. If some market maker learned more slowly than his peers, he would make losses at least until the next change in $p^{*}$. 